\title{
Comparison of Eight Detection Algorithms for the Quantification and Characterization of Mesoscale Eddies in the South China Sea
}

\author{
Zhan Lian, Baonan Sun, Zexun Wei, Yonggang Wang, And Xinyi Wang \\ Laboratory of Marine Science and Numerical Modeling, First Institute of Oceanography, State \\ Oceanic Administration, and Laboratory for Regional Oceanography and Numerical Modeling, \\ Qingdao National Laboratory for Marine Science and Technology, Qingdao, China.
}

(Manuscript received 17 November 2018, in final form 25 February 2019)

\begin{abstract}
Numerous oceanic mesoscale eddies occur in the South China Sea (SCS). The present study employs eight automatic eddy detection algorithms to identify these mesoscale eddies and compares the results. Eddy probabilities and areas detected by various algorithms differ substantially. Most regions of the SCS with a high discrepancy of eddy probabilities are those with few mesoscale eddies, except for the area west of the Luzon Strait, the area west of Luzon Island between $12^{\circ}$ and $17^{\circ} \mathrm{N}$, and the southernmost end of the SCS basin. They are primarily caused by strong interference, noncircular eddy shapes, and gentle sea level anomaly (SLA) gradients, respectively. The SLA, winding angle, and hybrid methods can easily detect the mesoscale eddies with wavelike features. The Okubo-Weiss (OW) and the spatially smoothed OW methods better identify grouping phenomena of mesoscale eddies in the SCS. Suggestions are presented on choosing suitable algorithms for studying mesoscale eddies in the SCS. No single algorithm is perfect for all research purposes. For different studies, the most suitable algorithm is different.
\end{abstract}

\section{Introduction}

Mesoscale eddies in the ocean are featured by swirling currents with a horizontal spatial scale of $10^{1}-10^{2} \mathrm{~km}$ and a temporal scale of $10^{1}-10^{2}$ days. The advent of high-accuracy satellite altimeter data has greatly improved our knowledge of mesoscale eddies in the past two decades (Chelton et al. 2011). To examine mesoscale eddies based on altimeter data, an eddy detection algorithm is necessary. The algorithms can be categorized into the following three classes: 1) those attempting to identify a rotational flow, 2) those attempting to identify closed sea level anomaly (SLA) contours, and 3 ) those combining the attributes of classes 1 and 2 . Although all algorithms can extract mesoscale eddies, different algorithms have different disadvantages. In the first category, the extra noise and arbitrary detection criteria may remove important physical information (Nencioli et al. 2010), whereas in the second and third categories, the results may be quite sensitive to the interval searching for closed SLA contours, especially in low-latitude areas. These disadvantages can induce significantly different results

\footnotetext{
Corresponding author: Zexun Wei,weizx@fio.org.cn
}

among different algorithms in certain areas (Souza et al. 2011).

The South China Sea (SCS) is one of the largest marginal seas of the western Pacific. It is connected to the Pacific Ocean through the Luzon Strait (LS), and the Kuroshio flows northward to the east of the LS most of the time in summer but often intrudes into the SCS in winter with a leaking path or loop path (Nan et al. 2015; Zhang et al. 2017). There are numerous oceanic mesoscale eddies in the SCS (Wang et al. 2003). These eddies play vital roles in many phenomena in the SCS, such as ocean circulation (Geng et al. 2016), ocean heat and salt transport (Chen et al. 2012), biogeochemical cycles (Guo et al. 2015), and deep-sea sediment distribution (Zhang et al. 2014). Therefore, quantifying and characterizing the mesoscale eddies in the SCS are of great significance.

Researchers have employed many algorithms to detect mesoscale eddies in the SCS. The algorithms in the first category are more commonly applied (Xiu et al. 2010; Nan et al. 2011b; Guo et al. 2015; Lin et al. 2015). The methods that fall into other categories have also been used (Wang et al. 2003; Chen et al. 2011; Yi et al. 2014). Using these algorithms, researchers have studied various features of these eddies, such as their 

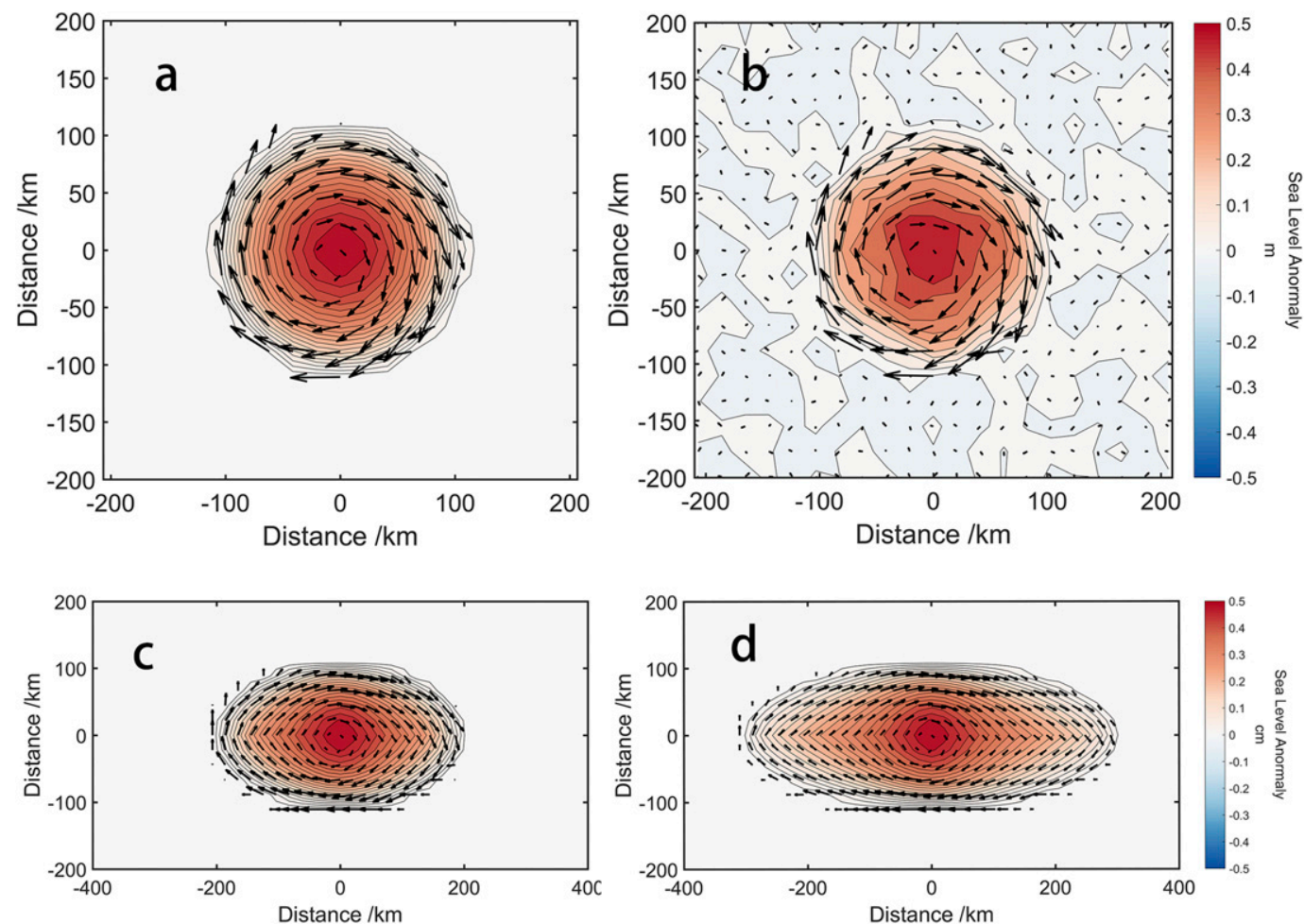

FIG. 1. Idealized eddies in the idealized experiments. (a) Round eddy without noise disturbance. (b) Round eddy with added noise at an SNR of 0.1. (c) Elliptical eddy with an ellipticity of 1.5. (d) Elliptical eddy with an ellipticity of 3.
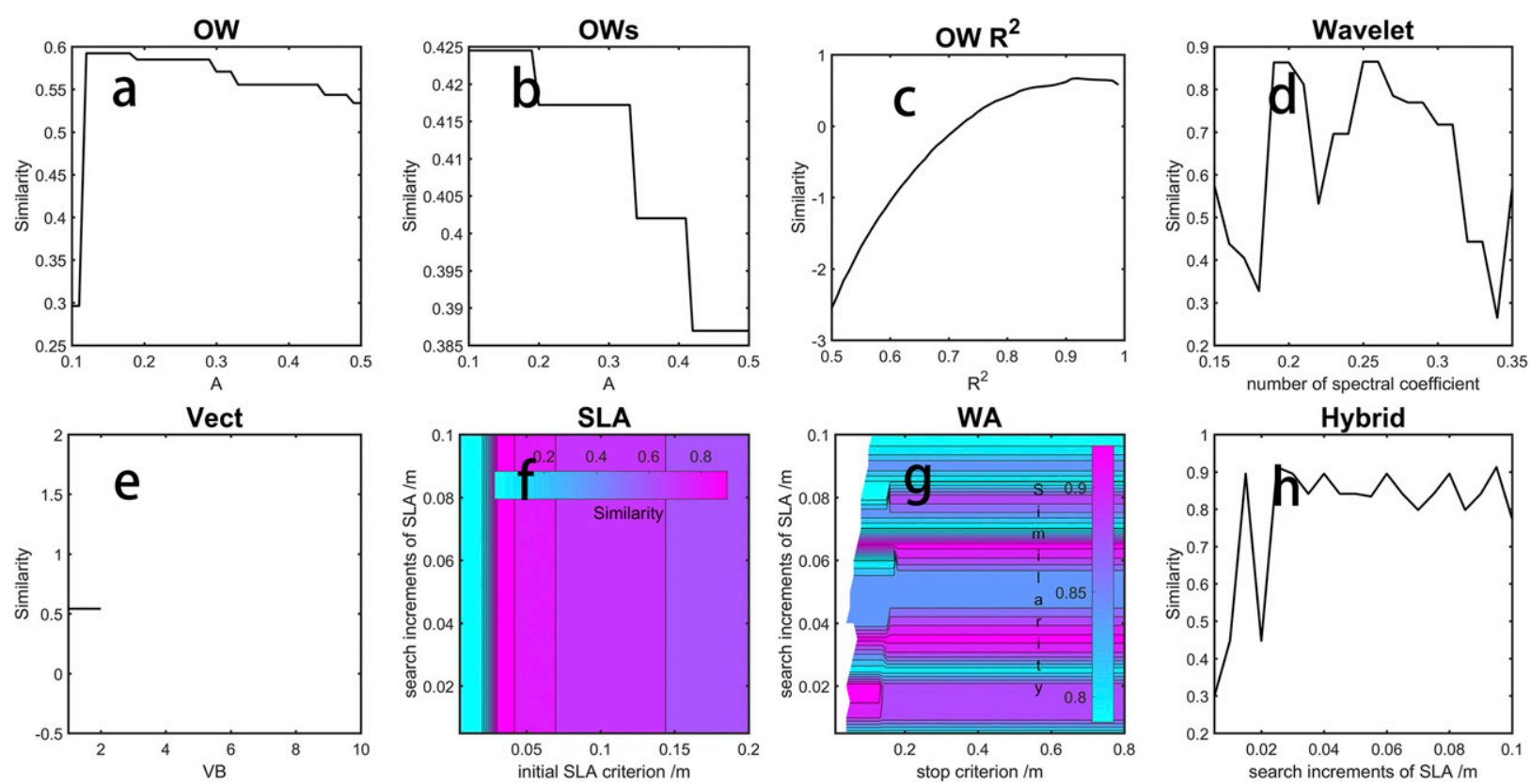

FIG. 2. Comparison of the parameters of the eight algorithms: (a) $A$ in the OW method, (b) $A$ in the OWs, (c) $R^{2}$ in the OW $R^{2}$ method, (d) number of spectral coefficients in the wavelet method, (e) VB in the vector method, (f) search increment of SLA and initial SLA criterion in the SLA method, (g) search increment of SLA and stop criterion in the WA method, and $(\mathrm{h})$ search increment of SLA in the hybrid method. 

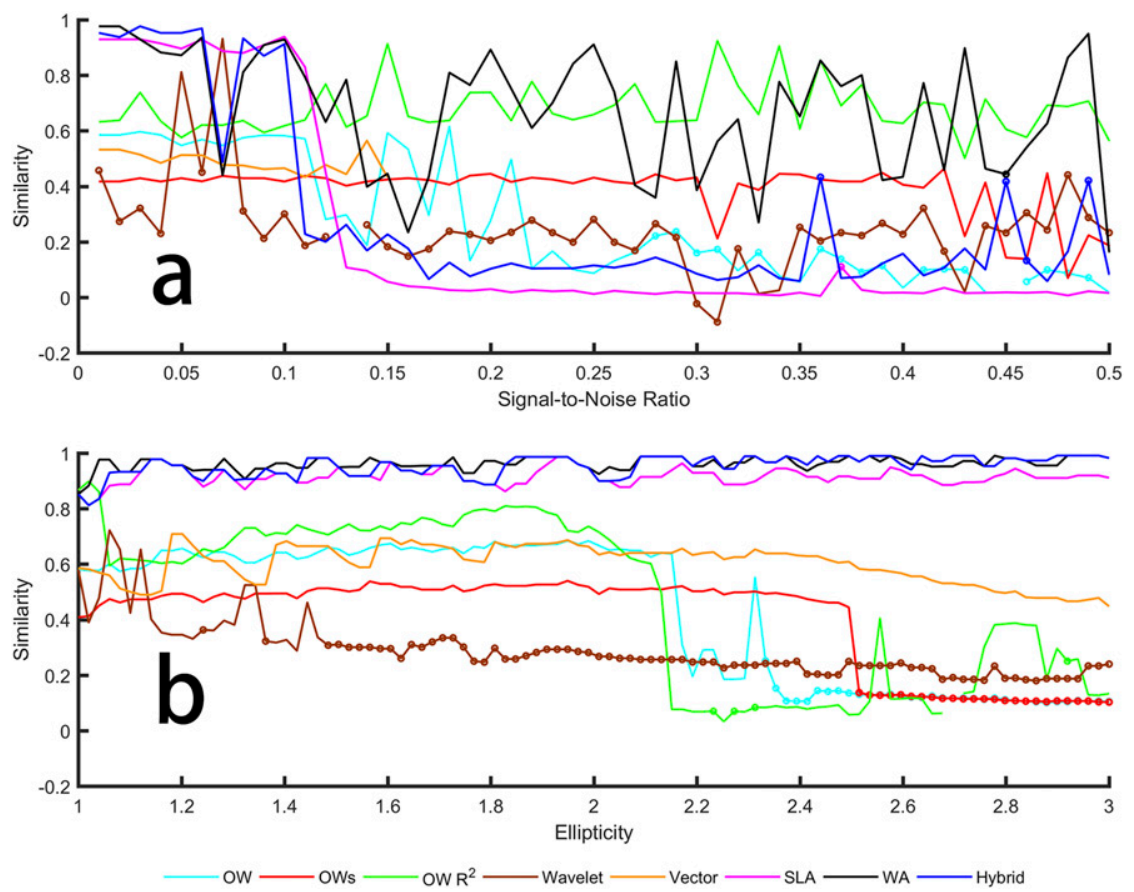

FIG. 3. Similarity between the eddies detected using the eight algorithms and the idealized eddies in the idealized experiments: (a) Exp A and (b) Exp B. The circles along each line indicate the presence of more than one identified eddy within the region occupied by the idealized eddy.

numbers, intensities, spatial distribution patterns, and vertical structures ( $\mathrm{Li}$ et al. 1998; Yuan et al. 2007; Wang et al. 2008; Nan et al. 2011a; Chu et al. 2014; Zhang et al. 2016). These studies indicate that there are several regions in the SCS where eddies are especially prevalent. The area west of Luzon Island (LI) and east of the Indo-China Peninsula are two typical examples.

In the results from different algorithms, obvious discrepancies are observed. For example, Xiu et al. (2010) estimated that the maximum eddy probability (which corresponds to the percentage of time that a given point is located within a mesoscale eddy) in the SCS is more than $30 \%$, although an estimate of more than $60 \%$ was given by Chen et al. (2011). The spatial distribution of eddy properties is also different in these studies. To the best of our knowledge, these discrepancies have not been previously estimated and compared. The present paper aims to assess the discrepancies of detected mesoscale eddy properties in the SCS among eight detection algorithms and examine the reasons causing the discrepancies.

This paper is organized as follows. The data and methods are described in section 2 . In section 3 , we first

TABLE 1. Mean properties of eddies in the SCS identified using different algorithms.

\begin{tabular}{lcccc}
\hline Detection algorithm & Probability $(\%)$ & Intensity $(\mathrm{cm})$ & Area $\left(10^{4} \mathrm{~km}^{2}\right)$ & Lifetime $($ days $)$ \\
\hline OW & 1.8 & 3.83 & 0.80 & 54.49 \\
OWs & 5.3 & 4.31 & 3.63 & 63.69 \\
OW $R^{2}$ & 0.3 & 12.80 & 2.26 & 39.04 \\
Wavelet & 0.8 & 7.51 & 2.31 & 44.23 \\
Vector & 1.3 & 2.38 & 8.51 & 44.32 \\
SLA & 6.0 & 6.86 & 9.34 & 55.26 \\
WA & 7.6 & 8.03 & 11.96 & 56.62 \\
Hybrid & 4.2 & 9.75 & 5.16 & 51.60 \\
Mean & 3.4 & 7.40 & 4.16 & 50.35 \\
Standard deviation & 2.8 & 3.74 & 0.80 & 9.12 \\
Coefficient of variation & 0.82 & 0.50 & & 0.18 \\
\hline
\end{tabular}




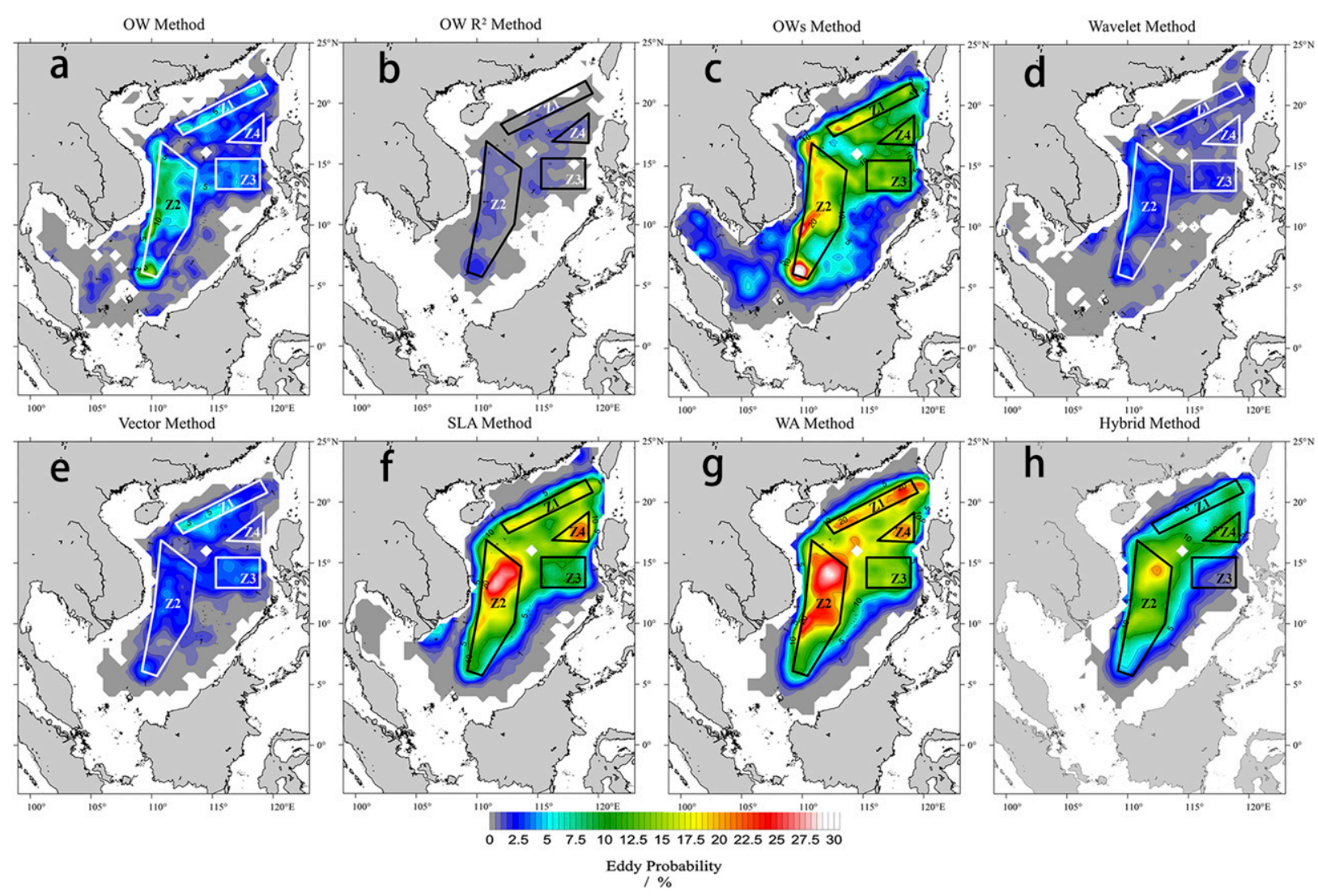

FIG. 4. Spatial distribution of the probability of eddies detected by the eight algorithms. The polygons indicate four eddy active zones in the SCS (Z1-Z4).

assess the ability of eight automatic eddy detection algorithms to detect idealized eddies; then we quantify the differences among the results of all algorithms in the SCS and analyze the causes. Finally, suggestions on eddy detection algorithm selection are presented in section 4 .

\section{Data and methods}

The present paper involves eight eddy detection algorithms. First, we used idealized eddies as criteria to determine critical parameters in all tested algorithms and assess the performance of these algorithms objectively. Then we used all algorithms to extract mesoscale eddies in the SCS from SLA and geostrophic velocity data observed from satellites.

\section{a. Idealized experiments}

The idealized experiments were set up as follows. In experiment A (Exp A), the radius of the idealized eddy was $100 \mathrm{~km}$ (Fig. 1a). To test the capacity of the different algorithms to resist disturbances, noise was deliberately added. We used the signal-to-noise ratio (SNR) to indicate the noise intensity (Figs. 1b). The magnitude of the noise gradually increased from 0 to a value equal to half of the maximum of the eddy intensity $(0<\mathrm{SNR}<$ $0.5)$. The purpose of experiment B (Exp B) is to assess the performance of different algorithms in identifying eddies with various shapes. An elliptical eddy was set as the idealized eddy. The ellipticity of this eddy was defined as the ratio of the major and minor axes. The minor axis of the elliptical eddy was $100 \mathrm{~km}$, and the ellipticity varied from 1 to 3 (1.5 in Fig. 1c and 3 in Fig. 1d). The purpose of experiment $\mathrm{C}(\operatorname{Exp} \mathrm{C})$ is to assess the performance of each algorithm in identifying an eddy that is near another eddy. The shapes of these two idealized eddies were the same as that in $\operatorname{Exp} \mathrm{A}$, and the distance between the two centers gradually decreased from 400 to $0 \mathrm{~km}$ with an increment of $20 \mathrm{~km}$. The SNR in this experiment was set to 0.1 .

The other options in these experiments are listed as follows. The spatial resolution of data in all experiments was $20 \mathrm{~km}$. The maximum SLA was located in the center of the eddy, and its magnitude was $0.5 \mathrm{~m}$. The SLA decreased from the center (maximum) to the edge of the eddy (zero) quadratically. The velocity was derived from the gradient of the SLA under geostrophic balance conditions. 


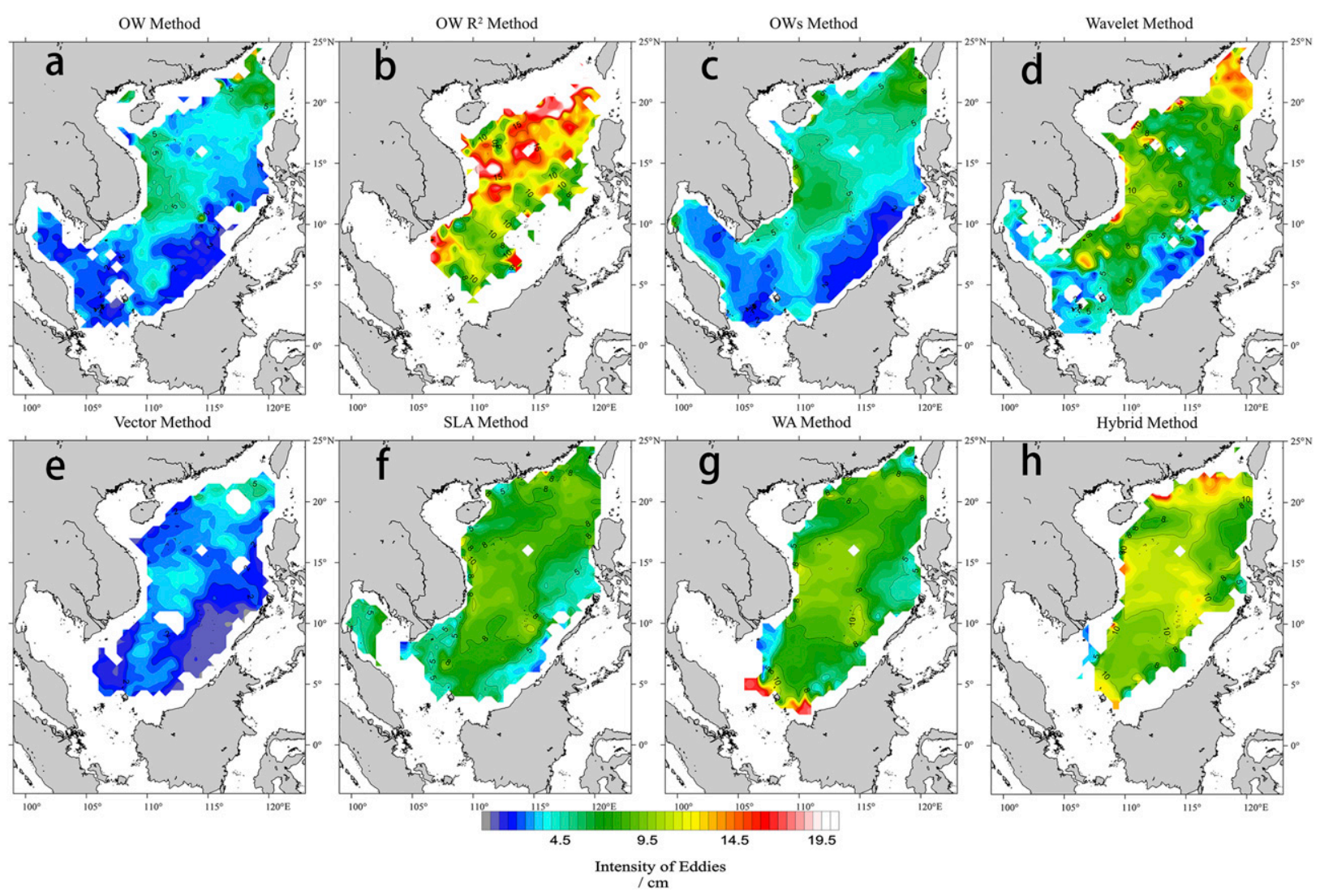

FIG. 5. As in Fig. 4, but for the intensity of eddies.

To represent the similarities between the idealized eddies and the detected eddies, the similarity (Si) was defined as follows:

$$
\mathrm{Si}=\mathrm{Si}_{1} \mathrm{Si}_{2} \mathrm{Si}_{3} \text {, }
$$

where $\mathrm{Si}_{1}=\overline{P_{d}} / \overline{P_{s}}, \mathrm{Si}_{2}=A_{d} / A_{s}, \overline{P_{d}}\left(\overline{P_{s}}\right)$, and $A_{d}\left(A A_{s}\right)$ represent the mean position and area of the detected eddy (idealized eddy), respectively; $\mathrm{Si}_{3}=N_{d} / N_{s}$, where $N_{d}$ is the number of eddies extracted, and $N_{s}$ is the actual number of eddies in these cases ( $N_{s}$ is 1 in Exp A and Exp B).

\section{b. Eddy detection algorithms}

We employed the original Okubo-Weiss (OW) method, the spatially smoothed OW (OWs) method, the OW $R^{2}$ method, the wavelet method, the rotating vector criterion method (called the vector method here), the SLA criterion method (called the SLA method here), the winding angle (WA) method, and the hybrid method. For the sake of simplicity, this paper only presents brief introductions for all tested algorithms and values of their crucial parameters used in this study. To select the most suitable crucial parameters for each algorithm, we used the round eddy in Exp A as a criterion, and the similarities were mean values corresponding to $0<\mathrm{SNR}<0.1$. We tried different values of the crucial parameters; the final parameters used in this paper were chosen to achieve the highest similarities. The introductions and crucial parameters of all methods are as follows.

\section{1) OW METHOD}

The OW parameter $W$ is developed to identify mesoscale eddies. This parameter is obtained by

$$
W=s_{n}^{2}+s_{s}^{2}-\omega^{2},
$$

where $\omega=\partial v / \partial x-\partial u / \partial y, s_{n}=\partial u / \partial x-\partial v / \partial y, s_{s}=\partial v / \partial x+$ $\partial u / \partial y$, and $u$ and $v$ are the zonal and meridional velocity components, respectively. An eddy is defined as the region where $W<-W_{0}$. The threshold $W_{0}$ is defined as $W_{0}=A \sigma W$, where $\sigma$ is the spatial standard deviation of $W$ with the same sign (Henson and Thomas 2008), and $A$ is a constant. The comparison between the idealized eddy and the identification suggest that $A=0.15$ is the best choice (Fig. 2a).

\section{2) OWS METHOD}

The noise in the resulting $W$ field makes it difficult to identify mesoscale eddies (Souza et al. 2011). To overcome 

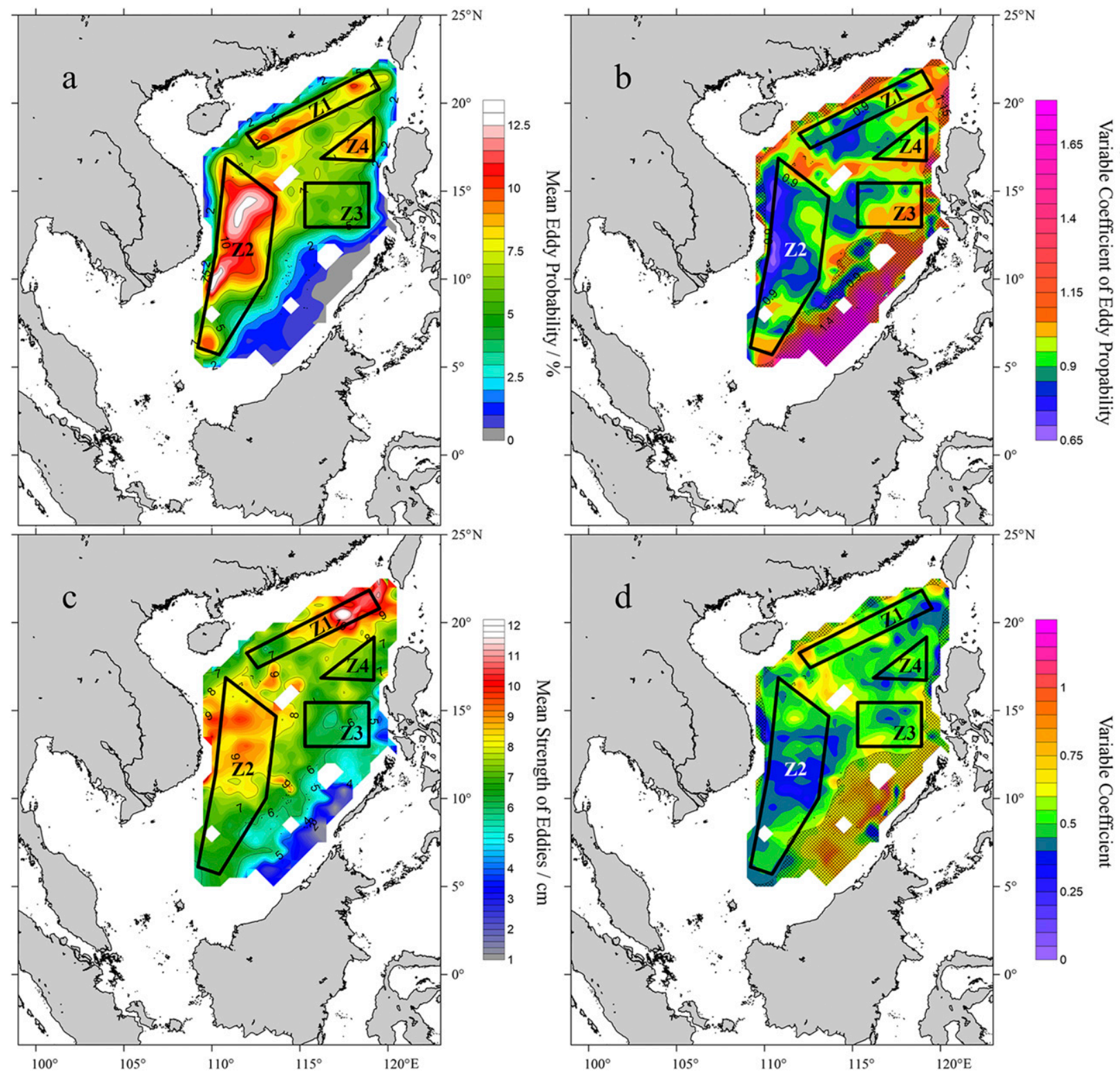

FIG. 6. (a) Spatial mean value and (b) coefficient of variation of the probability of eddies and (c) spatial mean value and (d) coefficient of variation of the intensity of eddies. The areas with water depths less than $200 \mathrm{~m}$ are excluded. The shaded areas in (b) and (d) indicate the areas with a probability of eddies that is less than half of the averaged value.

this issue, a low-pass spatial filter with a cutoff length scale of $100 \mathrm{~km}$ to the $W$ field is applied. The rest of the eddy detection method is as same as the OW method. Based on the comparison with the idealized eddy, we set $A$ to 0.15 in this method (Fig. 2b).

\section{3) OW $R^{2}$ METHOD}

The OW $R^{2}$ method was proposed by Williams et al. (2011) and Petersen et al. (2013). This approach judges the fitness of an eddy based on the similarity of its characteristics to an idealized Gaussian eddy, and the parameter $R^{2}$ describes this similarity. We selected a value of 0.9 for $R^{2}$ (Fig. 2c). Some numerical models used an idealized Gaussian initial condition to start the simulation (Geng et al. 2018). The OW $R^{2}$ method is suitable to detect the consequent eddy in this model, but it can also be tested with the satellite altimetry.

\section{4) WAVELET METHOD}

Following the wavelet method proposed by Doglioli et al. (2007), we first chose the optimal basis by minimizing the Shannon entropy of the $W$ field. The wavelets 

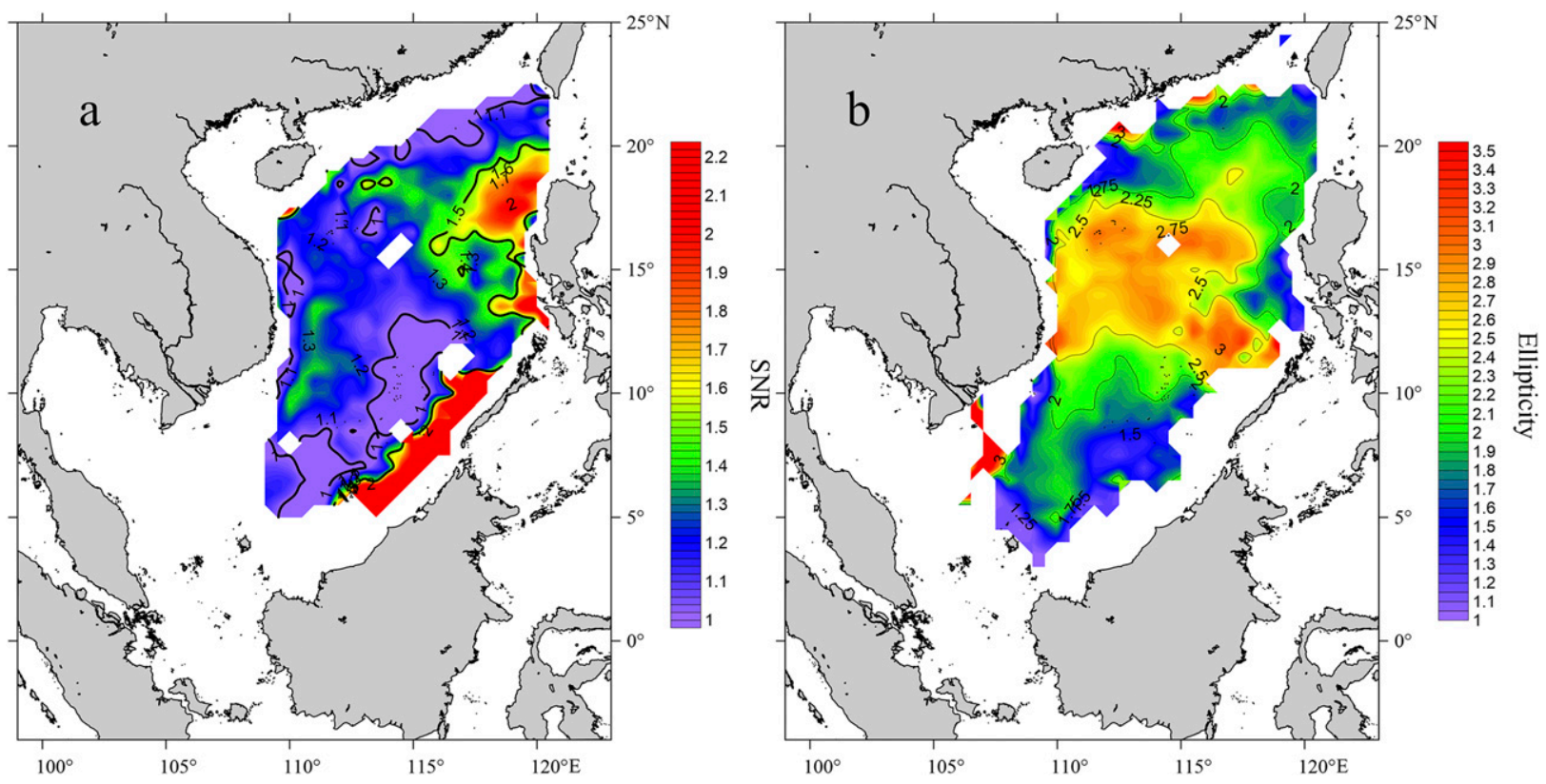

FIG. 7. (a) Ratio between the standard deviation of the SLA and mean eddy intensity and (b) mean eddy ellipticity from the hybrid method.

are then sorted as a function of their coefficients, and only the greatest $10 \%$ of the coefficients are retained as eddies. The regions with more than six grid cells in common are merged to eliminate filaments. The minimum number of grid cells of an eddy is set to 10 . The number of spectral coefficients retained for signal reconstruction is the critical parameter of this method (Doglioli et al. 2007). The analysis shows that 0.2 is the best value for this parameter (Fig. 2d).

\section{5) VECTOR METHOD}

Nencioli et al. (2010) proposed an approach for identifying eddies through directly applying a geometry criterion to currents. Four constraints are included in this method:

(i) Along with an east-west section, $v$ must reverse in sign across the eddy center. The magnitude of $v$ must increase away from the center.

(ii) Along with a north-south section, the changes in $u$ must be similar to those of $v$.

(iii) In a region with a dimension of VB grid points, the velocity magnitude has a local minimum at the eddy center.

(iv) Around the eddy center, the directions of the velocity vectors must change with a constant sense of rotation.

The points that meet the conditions listed above are identified as eddy centers. The closed streamline around the center of an eddy with the maximum current speed is defined as the boundary of that eddy. The most important parameter for detecting the eddies is VB. Based on our analysis (Fig. 2e), we set VB to 1.

\section{6) SLA METHOD}

Chelton et al. (2011) presented an eddy identification approach that employs an SLA criterion. From the maximum (minimum) data, we search the closed contours of the SLA for anticyclonic (cyclonic) eddies. The searching starts with an initial SLA criterion, and the searching range changes by an interval. The connected regions contain at least 8 grids but fewer than 1000 grids. Anticyclones (cyclones) contain at least one local SLA maximum (minimum). The distance between any pair of points within the connected region must be less than $800 \mathrm{~km}$. As shown in the comparison with the idealized eddy (Fig. 2f), the detections are quite sensitive to the initial SLA criterion but not to the SLA search interval. These two parameters were set to 0.02 and $0.035 \mathrm{~m}$.

\section{7) WA METHOD}

The WA detection algorithm uses a closed SLA contour as a detection criterion (Sadarjoen and Post 2000). There are many versions of this algorithm. In this study, we used the version developed by Chaigneau et al. (2009), which optimizes the computational time compared to the original version (Chaigneau et al. 2008). The cyclone (anticyclone) detection algorithm involves, first, searching for eddy centers that are local SLA minima (maxima) within a moving window of $4 \times 4$ grid 


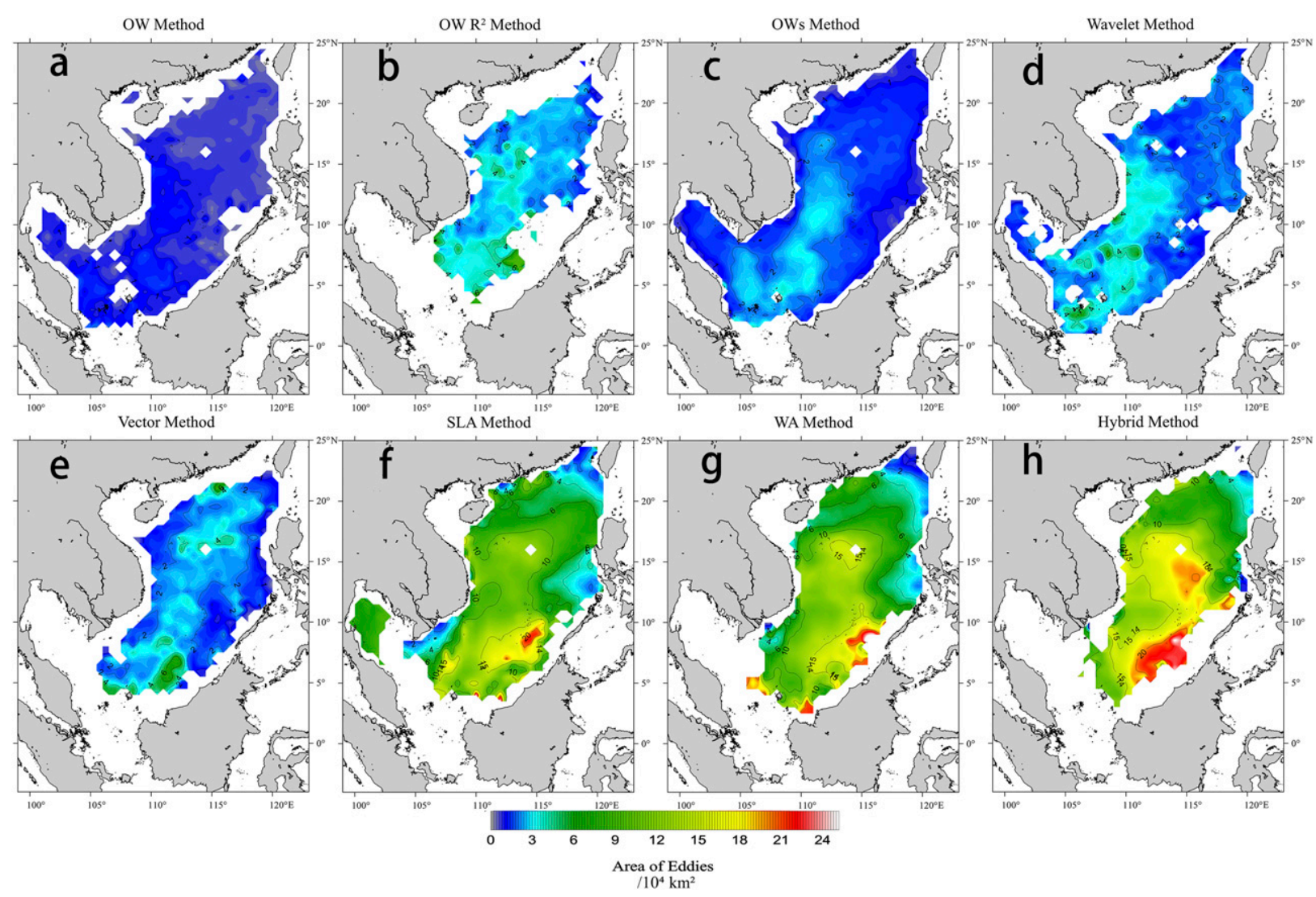

FIG. 8. As in Fig. 4, but for the areas of eddies.

points. Then, for each possible cyclonic (anticyclonic) center, the algorithm searches for closed SLA contours with an increment (decrement). The outermost closed SLA contour that contains only the considered center corresponds to the edge of the eddy. The SLA search increment and the SLA stopping criterion (indicating the maximum range for finding the closed contour line) were set to 0.035 and $0.55 \mathrm{~m}$, respectively (Fig. $2 \mathrm{~g}$ ).

\section{8) HYBRID METHOD}

The hybrid method employs the integrated criteria of the OW method and the SLA method (Yi et al. 2014). In this algorithm, we use the OW method to detect the cores of eddies and the SLA method to extract the eddy edge. The optimal value of the SLA search increment was $0.01 \mathrm{~m}$ according to the comparison with the idealized eddy (Fig. 2h).

Algorithms in this study belong to different categories. An eddy is defined as a region where rotational circulation dominates by the OW family of methods (the OW, OWs, and OW $R^{2}$ methods), the wavelet method, and the vector method, and all these methods can be categorized as class 1 algorithms. In contrast, in the SLA and WA methods, a closed SLA contour line is used as the criterion, so these methods belong to class 2 algorithms. All the above methods define the eddy center and boundary via the same procedure except for the hybrid method.

\section{c. Satellite data}

The SLA and geostrophic velocity data were obtained from the Archiving, Validation, and Interpretation of Satellite Oceanographic Data (AVISO) program, and the 2014 version of the Data Unification and Altimeter Combination System (DUACS; version 15.0) data was used. These data have a spatial resolution of $1 / 4^{\circ}$ and a 1-day temporal resolution. Data covering the period from January 1993 to September 2015 were employed in the analyses presented here.

\section{d. Eddy intensity}

The eddy intensity was defined as the difference between the extremum of the SLA and the mean value within the eddy.

\section{e. Eddy-tracking algorithm}

Following the approach proposed by Nencioli et al. (2010), after detecting the eddy centers, the eddy tracks 


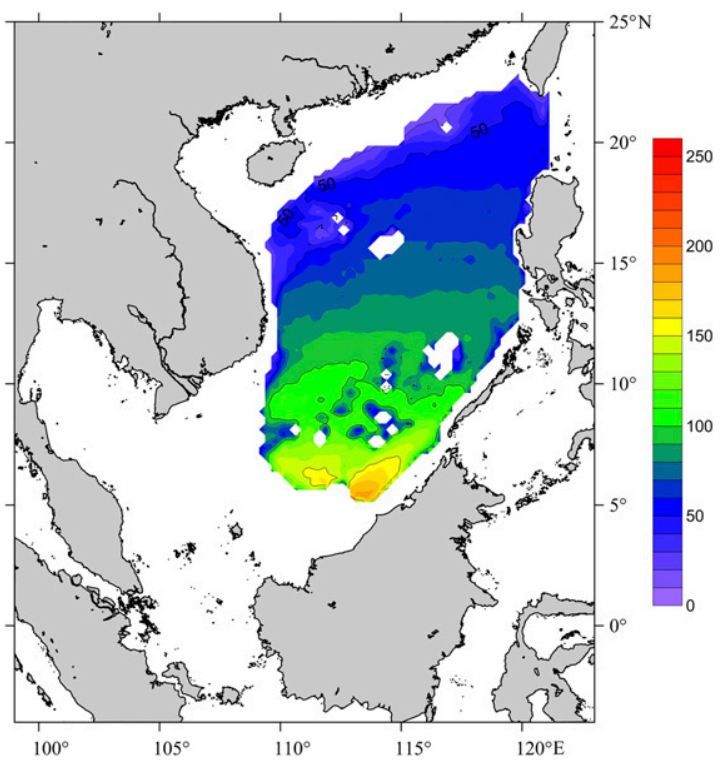

FIG. 9. Spatial distribution of the first baroclinic Rossby deformation radius in the SCS. The areas with water depths less than $200 \mathrm{~m}$ are excluded.

are determined by comparing the positions of the centers at successive time steps. The track of a given eddy at time step $t$ is updated by searching for eddy centers of the same type (i.e., cyclonic or anticyclonic) at time $t+1$ within a search zone with a radius of $100 \mathrm{~km}$. The present study used daily data, so this search zone can include eddies with speeds less than $1.2 \mathrm{~m} \mathrm{~s}^{-1}$ (radius per day). According to Chen et al. (2011), this threshold speed is larger than the advection speed of most eddies in the SCS; therefore, we think the selection of search radius is reasonable.

Eddies with lifetimes shorter than 30 days are removed from the analysis.

\section{Results and discussion}

The present paper involves eight eddy detection algorithms. First, we assessed their performance through idealized experiments. All eddy identifications in the SCS were then compared and analyzed.

\section{a. The idealized experiments}

The identifications from various methods in the idealized experiments are different and changing with different experiment settings. Some identifications under specific settings are shown in appendix A. To assess the performances of all methods under all experiment settings, Fig. 3a shows the similarities between the detected and idealized eddies in Exp A. When the noise intensity

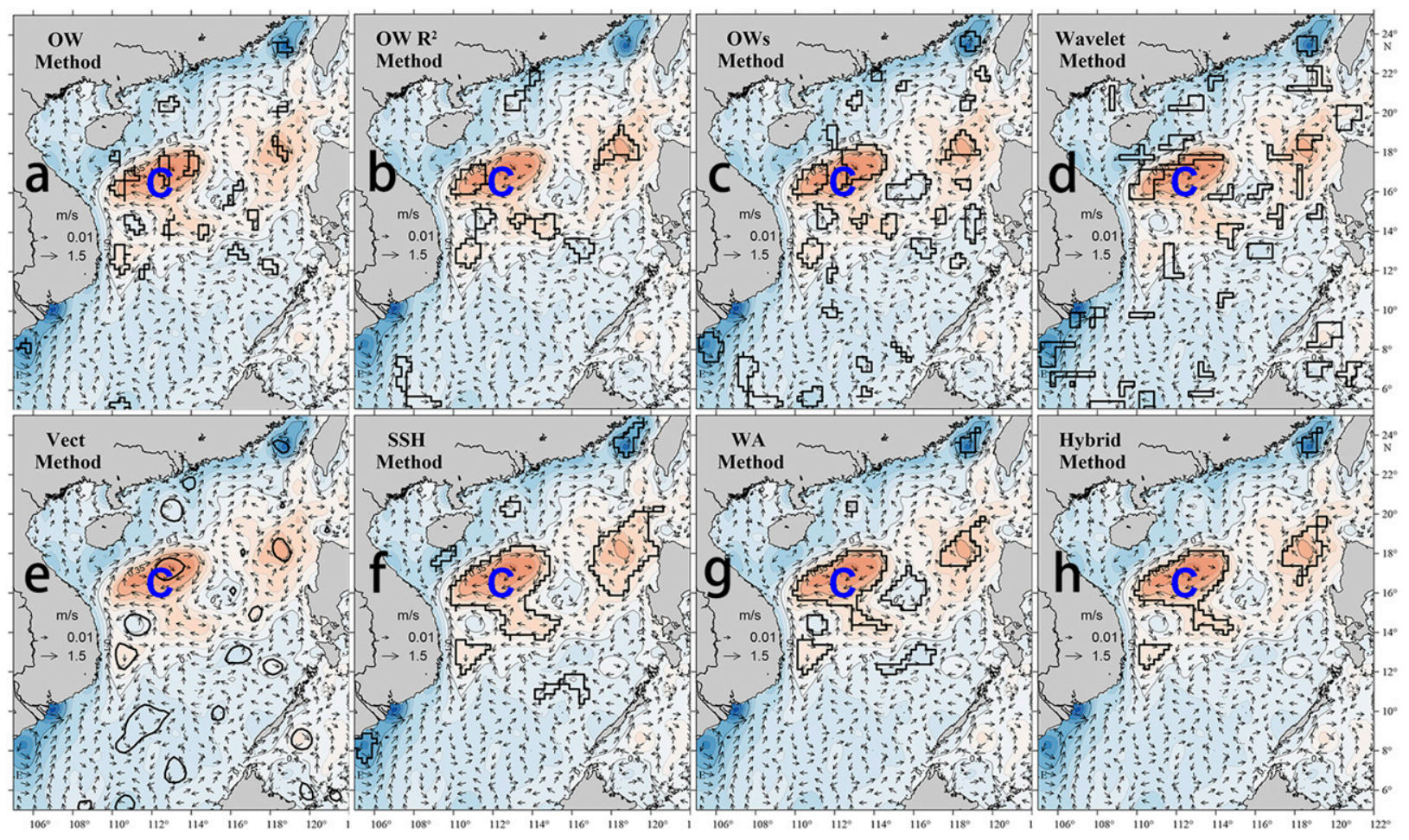

FIG. 10. Detected results for 10 Aug 2010 for all tested algorithms. The color contours indicate SLA in the SCS, the vectors indicate ocean current, the black polylines show the eddy edges detected by all algorithms, and the blue letter "C" in the ocean basin indicates the target eddy in this case study. 


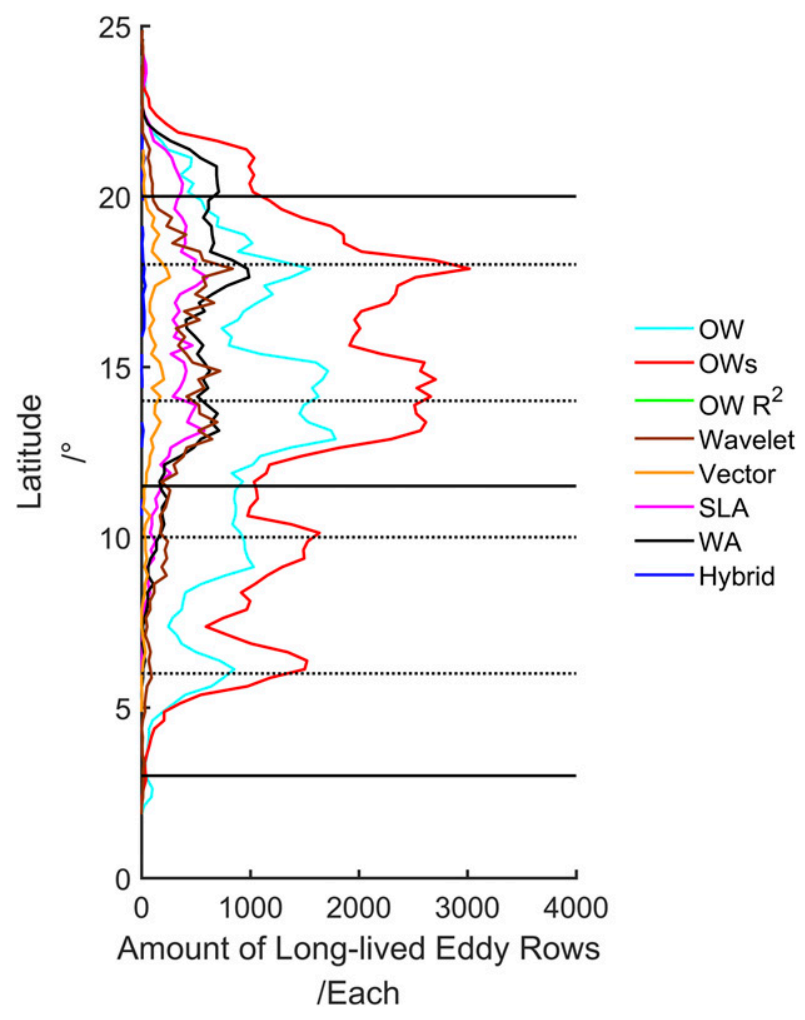

FIG. 11. The meridional distribution of the number of eddy trains.

is low, the similarities from class 2 and 3 methods (the WA, SLA, and hybrid methods) are more acceptable than those from class 1 . However, with the increase in the SNR, the performances from class 2 and 3 methods are either substantially deteriorated (the SLA and hybrid methods) or not stable (the WA method). Unlike the above methods, for the class 1 methods, the performances of some methods (the OW, OW $R^{2}$, and OWs methods) are stable and high-under-high SNR, whereas the wavelet method tends to isolate too many eddies (more than 1) and the vector method cannot identify an eddy. The missing identification of the vector method is because that the constraint used in this method requires that the change near the minimum velocity in the center of an eddy is monotonic. However, because of the quadratic changes of SLA in the experiment, the magnitude of the velocity in the eddy center is low; thus, this constraint tends to be not satisfied because of noise.

The situation is quite different for Exp B (Fig. 3b). First, for an eddy with a noncircular shape, the accuracies of class 2 and 3 methods are higher than those of class 1 because the class 1 methods are only sensitive to the core of the eddy; thus, they tend to underestimate the eddy area. This performance is induced by the different definition of the eddy area. For class 1, they choose the maximum swirling velocity as the edge of an eddy. Instead, the other methods choose the outmost close SLA contour. For an elliptical eddy, the rotatability of currents is only significant in the core. The class 1 methods tend to neglect the peripheral eddy area (Fig. A2). This underestimation increases as the ellipticity increases. Additionally, under high ellipticity, deficiencies of the class 1 methods are also caused by the excessive extraction of eddies.

In Exp C, before the two eddies are identified as one, the final distances identified between the two centers among all methods are 60 (the OW method), 80 (the OWs method), 100 (the OW $R^{2}$ method), 110 (the wavelet method), 120 (the vector method), 240 (the SLA method), 240 (the WA method), and $260 \mathrm{~km}$ (the hybrid method). Considering that the radii of these idealized eddies are both $100 \mathrm{~km}$, the results from the class 2 and 3 methods are more acceptable.

\section{b. SCS}

Table 1 presents the mean probabilities, intensities, areas, and lifetimes of the extracted eddies. The differences of some properties are substantial. The eddy probabilities from the OW $R^{2}$ method are the lowest, although this method yields the highest eddy intensities, indicating that this method is not suitable for studying the mean eddy properties in the SCS. The eddy probabilities obtained from the wavelet, vector, and OW

TABLE C1. Parameters selected for the tested algorithms.

\begin{tabular}{|c|c|c|c|c|c|}
\hline & Exp PA & Exp PB & Exp PC & Exp PD & Exp PE \\
\hline OW & $A=-0.15$ & $A=-0.15$ & $A=-0.14$ & $A=-0.16$ & $A=-0.17$ \\
\hline OW $R^{2}$ & $R^{2}=0.9$ & $R^{2}=0.91$ & $R^{2}=0.92$ & $R^{2}=0.89$ & $R^{2}=0.88$ \\
\hline OWs & $A=-0.15$ & $A=-0.15$ & $A=-0.14$ & $A=-0.16$ & $A=-0.17$ \\
\hline Wavelet & Factor $=0.20$ & Factor $=0.27$ & Factor $=0.25$ & Factor $=0.22$ & Factor $=0.28$ \\
\hline Vector & $\mathrm{VB}=1$ & $\mathrm{VB}=1$ & $\mathrm{VB}=1$ & $\mathrm{VB}=1$ & $\mathrm{VB}=1$ \\
\hline SLA & $\begin{aligned} \text { eddy_crit } & =0.035 \mathrm{~m} \\
\text { d_ssh } & =0.02 \mathrm{~m}\end{aligned}$ & $\begin{aligned} \text { eddy_crit } & =0.035 \mathrm{~m} \\
\text { d_ssh } & =0.02 \mathrm{~m}\end{aligned}$ & $\begin{aligned} \text { eddy_crit } & =0.01 \mathrm{~m} \\
\text { d_ssh } & =0.01 \mathrm{~m}\end{aligned}$ & $\begin{aligned} \text { eddy_crit } & =0.02 \mathrm{~m} \\
\text { d_ssh } & =0.005 \mathrm{~m}\end{aligned}$ & $\begin{aligned} \text { eddy_crit } & =0.05 \mathrm{~m} \\
\text { d_ssh } & =0.0025 \mathrm{~m}\end{aligned}$ \\
\hline WA & $\begin{aligned} \text { stop_crit } & =0.55 \mathrm{~m} \\
\text { d_ssh } & =0.035 \mathrm{~m}\end{aligned}$ & $\begin{aligned} \text { stop_crit } & =0.55 \mathrm{~m} \\
\text { d_ssh } & =0.015 \mathrm{~m}\end{aligned}$ & $\begin{aligned} \text { stop_crit } & =0.7 \mathrm{~m} \\
\text { d_ssh } & =0.01 \mathrm{~m}\end{aligned}$ & $\begin{aligned} \text { stop_crit } & =0.7 \mathrm{~m} \\
\text { d_ssh } & =0.005 \mathrm{~m}\end{aligned}$ & $\begin{aligned} \text { stop_crit } & =1 \mathrm{~m} \\
\text { d_ssh } & =0.0025 \mathrm{~m}\end{aligned}$ \\
\hline Mixed & d_ssh $=0.025 \mathrm{~m}$ & d_ssh $=0.025 \mathrm{~m}$ & d_ssh $=0.01 \mathrm{~m}$ & d_ssh $=0.005 \mathrm{~m}$ & d_ssh $=0.0025 \mathrm{~m}$ \\
\hline
\end{tabular}



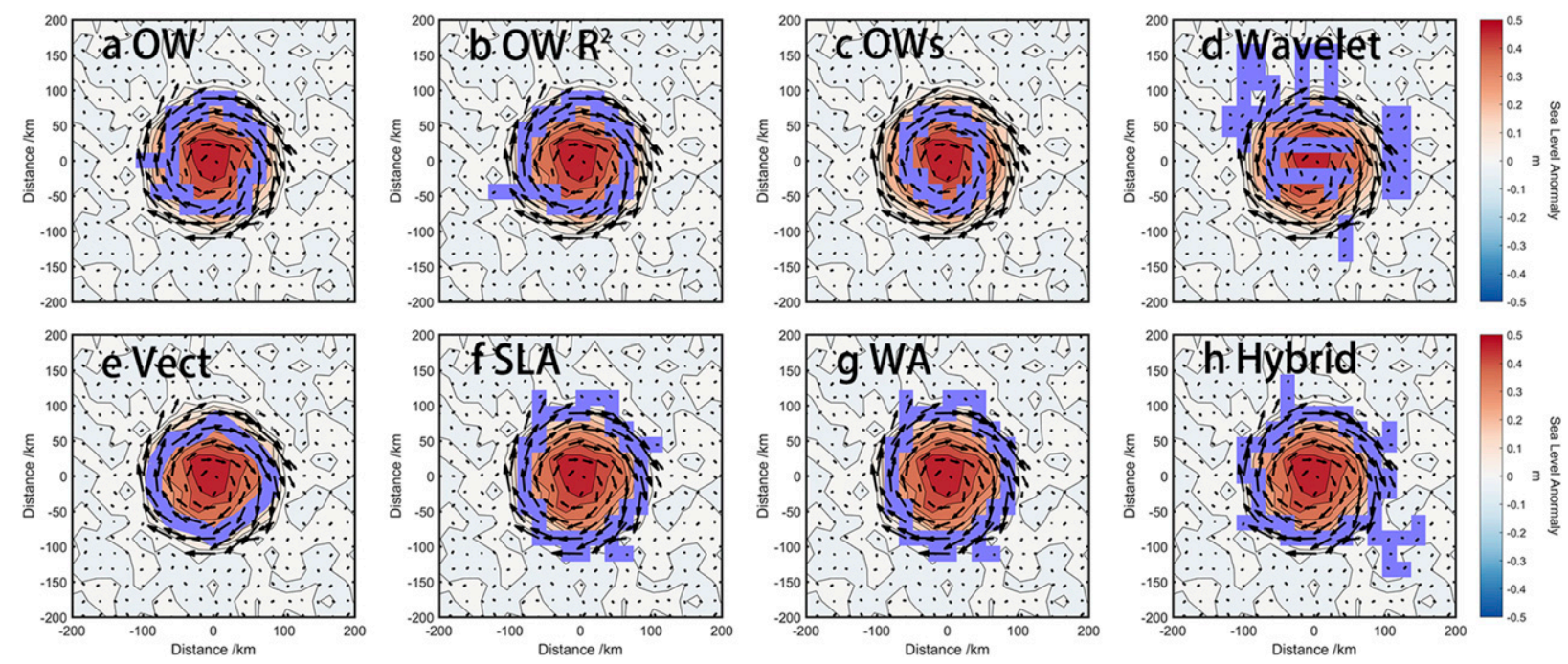

FIG. A1. Eddy identification in Exp A $(\mathrm{SNR}=0.1)$. The purple polylines indicate the data grids composing the detected eddy edges.

methods are also lower than those of the other methods. The results of the idealized experiments indicate that these deviations might be related to the tendency of excessive detection, the high number of missing eddies, and small size of isolated areas. The mean eddy areas and intensities from the second and third categories of methods are greater than those of the first category. The fact that the first category and the others detect the eddy edge via different definitions (the maximum swirling velocity for the former and the maximum SLA isoline for the later) may lead to this phenomenon. We also calculate the mean value of all algorithms, the standard deviation, and the coefficient of variation for each element. The coefficient of variation is a nondimensional number that is defined as the standard deviation divided by the mean value. A greater coefficient of variation indicates that the discrepancy is more significant. The coefficients of variation of the eddy probability and area are the highest, and the second highest value is for eddy intensity. These results suggest that in studies of the areas, intensities, and probabilities of eddies, the eddy detection algorithm should be chosen with care. However, the difference in eddy lifetimes is not significant.

Figure 4 presents the spatial distributions of the eddy probabilities on a $0.5^{\circ} \times 0.5^{\circ}$ grid. The results show that although the spatial patterns of the eddy probabilities from all algorithms are similar, the difference is also obvious. Regarding to the similar distribution, four active zones of eddies are observed in the SCS basin. The northern (Z1) and eastern (Z2) zones at the edge of the SCS basin and the zone (Z3) traversing the SCS basin at $13^{\circ} \mathrm{N}$ are evident, and the zone extending from the west coast of LI (Z4) at approximately $16^{\circ}-17^{\circ} \mathrm{N}$ is less evident. The separation between $\mathrm{Z} 1$ and $\mathrm{Z} 4$ is ambiguous in some methods (e.g., the OW $R^{2}$, vector, and WA methods). Except for the similar pattern, an obvious difference is observed in the maximum probability among different algorithms. In a real ocean, the definition

TABLE C2. Mean properties of eddies identified in the SCS using different algorithms in Exp PB.

\begin{tabular}{lcccc}
\hline \hline Detection algorithm & Probability $(\%)$ & Intensity $(\mathrm{cm})$ & Area $\left(10^{4} \mathrm{~km}^{2}\right)$ & Lifetime $($ days $)$ \\
\hline OW & 1.8 & 3.83 & 0.80 & 54.49 \\
OWs & 5.3 & 4.31 & 2.93 & 63.69 \\
OW $R^{2}$ & 0.3 & 12.04 & 2.37 & 38.97 \\
Wavelet & 0.8 & 7.6 & 2.31 & 41.90 \\
Vector & 1.3 & 2.38 & 8.85 & 44.32 \\
SLA & 6.0 & 7.08 & 6.68 & 54.56 \\
WA & 7.6 & 5.85 & 11.96 & 54.27 \\
Hybrid & 4.2 & 9.75 & 4.70 & 51.60 \\
Mean & 3.5 & 7.14 & 4.01 & 50.47 \\
Standard deviation & 3.0 & 3.21 & 0.85 & 8.17 \\
Coefficient of variation & 0.84 & 0.49 & & 0.16 \\
\hline
\end{tabular}


TABle C3. As in Table C2, but for Exp PC.

\begin{tabular}{|c|c|c|c|c|}
\hline Detection algorithm & Probability (\%) & Intensity (cm) & Area $\left(10^{4} \mathrm{~km}^{2}\right)$ & Lifetime (days) \\
\hline OW & 1.9 & 3.90 & 0.81 & 54.94 \\
\hline OWs & 5.4 & 4.35 & 1.71 & 64.06 \\
\hline OW $R^{2}$ & 0.4 & 12.85 & 2.46 & 37.82 \\
\hline Wavelet & 0.8 & 7.68 & 2.27 & 43.05 \\
\hline Vector & 1.3 & 2.38 & 2.31 & 44.32 \\
\hline SLA & 7.0 & 6.92 & 8.78 & 52.37 \\
\hline WA & 8.6 & 5.52 & 6.25 & 54.99 \\
\hline Hybrid & 4.4 & 9.02 & 11.02 & 50.69 \\
\hline Mean & 3.7 & 6.57 & 4.45 & 50.28 \\
\hline Standard deviation & 3.1 & 3.32 & 3.76 & 8.29 \\
\hline Coefficient of variation & 0.84 & 0.51 & 0.85 & 0.16 \\
\hline
\end{tabular}

of an eddy is arbitrary and is too subjective to describe the maximum probability by only one number. It is more reasonable to provide a range of maximum probability through ensemble mean, which is $18 \% \pm 11 \%$ according to the mean value and standard deviation among all algorithms. Next, the other characteristics of discrepancy are analyzed combined with other eddy properties.

Figure 5 shows the spatial patterns of the mean intensities of eddies in the SCS. Most algorithms indicate that the eddies occurring to the west of the LS and east of the Indo-China Peninsula display the highest intensities except for the OW $R^{2}$ and hybrid methods. The results from the OW $R^{2}$ method display no clear spatial pattern, whereas the results of the hybrid method show that the high-intensity southern core shifts to the eastern border of the SCS basin.

To further quantify the differences among the various methods, we calculate the mean values and coefficients of variation of all methods for the eddy probabilities and intensities (Fig. 6). The main regions with high coefficients of variation present low eddy probabilities (the areas with contour shading in Figs. $6 b$ and $6 d$ ), except for three places.

The first location is located at the easternmost end of $\mathrm{Z} 1$, and the differences are probably related to the high
SNR. Figure 7a shows the ratio between the standard deviations of the mesoscale SLA and the mean eddy intensities among all algorithms. A higher ratio indicates a greater disturbance (higher SNR). The maximum ratio emerges to the west of LS. This value can be induced by the intrusion of the Kuroshio into the SCS through the LS; thus, the circulation in this area presents a high magnitude and a complex structure. Therefore, significant interference effects are expected there.

The second location lies at the end of $\mathrm{Z} 3$ and Z4, which is close to the west side of LI. The mean eddy intensities are low in this area (Fig. 6c); thus, a high SNR is also expected here. Hence, an obvious difference is likely to appear here. In addition, the spatial pattern of eddy shape also plays a role in these results. Figure $7 \mathrm{~b}$ presents the eddy ellipticity inferred from the results of the hybrid method. The eddy shape shows a high ellipticity in these areas, which can also cause perturbations in some methods.

The last location lies in the southernmost end of the SCS basin. High eddy probabilities arise in this area only in the results of the first category algorithms. The concept of geostrophic balance can explain this discrepancy. For geostrophic motion, the velocity is proportional to the product of the inverse of the Coriolis parameter $f$
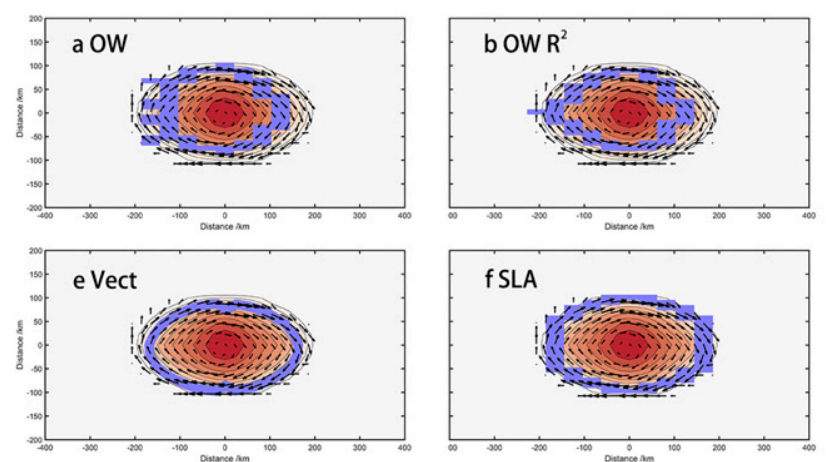
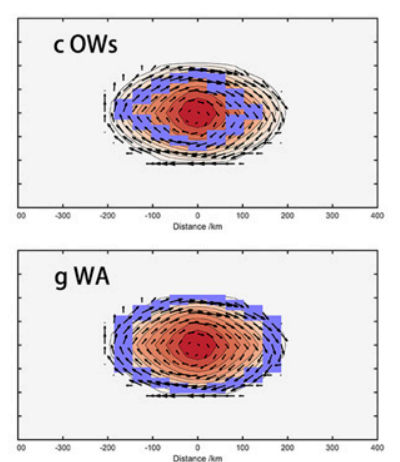
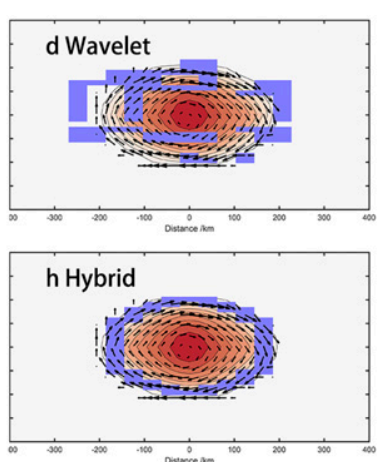

FIG. A2. Eddy identification in Exp B (ellipticity =1.5). The purple polylines indicate the data grids composing the detected eddy edges. 

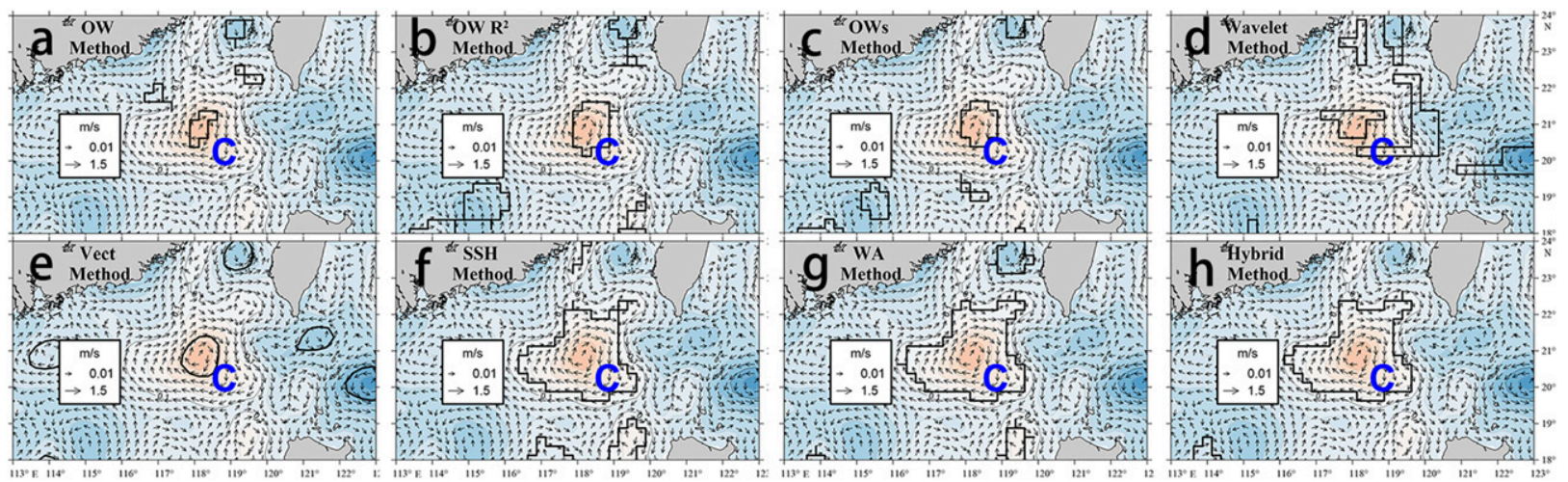

FIG. B1. Detected results for 10 Sep 1994 for all tested algorithms. The color contours indicate SLA in the SCS, the vectors indicate ocean current, the black polylines show the eddy edges detected by all algorithms, and the blue letter " $\mathrm{C}$ " in the ocean basin indicates the target eddy in this case study.

and the pressure gradient. At the southernmost end of the SCS basin, $f$ is the lowest. Thus, eddies with weaker SLA gradients develop under the same velocity magnitude. To detect such eddies, methods in the second and third detection algorithm classes should be employed with finer SLA search increments. However, the results from the first detection algorithm category are unaffected.

The spatial distribution of the area of eddies is presented in Fig. 8. The western part of the SCS basin features larger eddies than those in the eastern part, according to the results of the first category of algorithms. However, the opposite results are obtained for the SLA, WA, and hybrid methods. The Rossby wave theory can explain this difference. As noted by Chelton et al. (2011), the eddy radius is closely related to the first baroclinic Rossby deformation radius $R_{n}$ with $n=1$.
Under the Wentzel-Kramers-Brillouin (WKB) approximation, $R_{1}$ is given by (Chelton et al. 1998)

$$
R_{n}=\frac{1}{|f| n \pi} \int_{-H}^{0} N(z) d z, \quad n=1,
$$

where $N(z)$ is the Brunt-Väisälä frequency, and $H$ is the water depth. Using the World Ocean Atlas 2013, we calculated the spatial pattern of $R_{1}$ in the SCS (Fig. 9). The core of the area with greater $R_{1}$ values is clearly biased toward the eastern side of the SCS. The closer the eddy radius is to the Rossby deformation radius, the tighter the relationship between these two phenomena (eddy and Rossby wave) must be and the more wavelike this eddy is. The spatial pattern of eddy area identified by the SLA, WA, and hybrid methods is similar with that of $R_{1}$. These methods therefore may detect eddies with

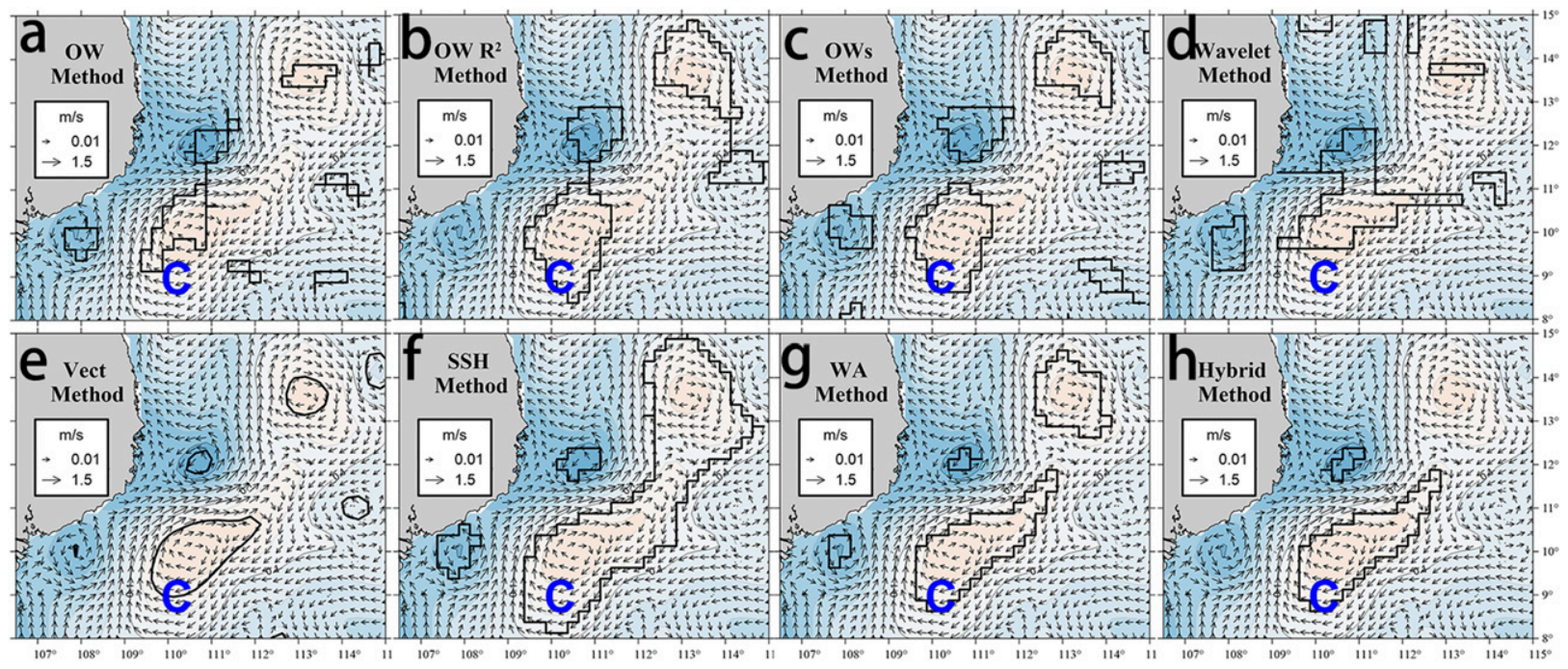

FIG. B2. As in Fig. B1, but for 5 Sep 2007 

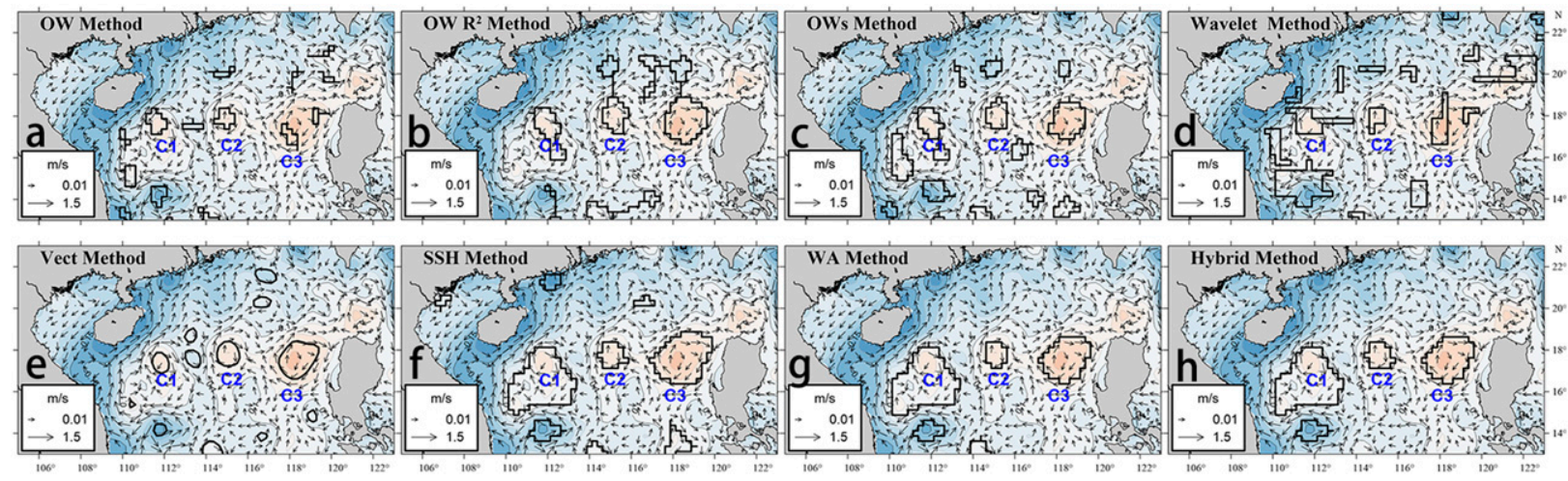

FIG. B3. As in Fig. B1, but for 22 Aug 2007 and the blue letters "C1," "C2," and "C 3 " in the ocean basin indicates the target eddies in this case study.

more wavelike features. Conversely, the other methods are more sensitive to swirling currents, which is another typical feature of eddies. This is another interpretation of the discrepancy among the tested algorithms.

To further delineate the distinctions among the detections from all the algorithms, we also carried out a case study on an anticyclonic eddy emerging to the east of Hainan Island. The amplitude of this eddy was exceptionally strong during July and August 2010 according to observations (Chu et al. 2014). The detections from all algorithms on 10 August 2010 are presented in Fig. 10. The structures detected by the OW family of methods tend to be located in the area where there is a high shear current. The vector method detects the eddy located close to the geometrical center of the swirling current. This case study demonstrates the differences in the principles used in each algorithm vividly. More case studies are shown in appendix B.

The results of the various detection algorithms do not display obvious differences in the inferred eddy lifetimes. Therefore, we do not analyze the spatial distribution of this parameter in detail in this paper.

Last, to assess the effect of critical parameters in each algorithm on the final comparison, we designed a parallel experiment (as shown in appendix C). The result confirms that the discrepancy among all algorithms is not parameter dependent and should be explained by the properties of mesoscale eddies in the SCS.

\section{c. Application}

In addition to examining the spatial distribution of eddy properties in the SCS, the comparison of different eddy detection algorithms can also shed light on other important issues.

The first issue is about the source of eddies near the LS. Some investigators have suggested that eddies can travel across the LS with the Kuroshio (Sheu et al. 2010), but the amount of these eddies is not big (Chen et al. 2011). To verify the discrepancies caused by the detection algorithms, we use each algorithm to extract the eddies passing through the LS. The results indicate that few long-lived eddies can traverse the western Pacific Ocean and intrude into the SCS; 0, 2, 0, 2, 0, 1, 2, and 0 such eddies are identified using the OW, OWs, OW $R^{2}$, wavelet, vector, SLA, WA, and hybrid methods, respectively. This result suggests that the choice of eddy detection algorithm is not crucial to this issue. The main
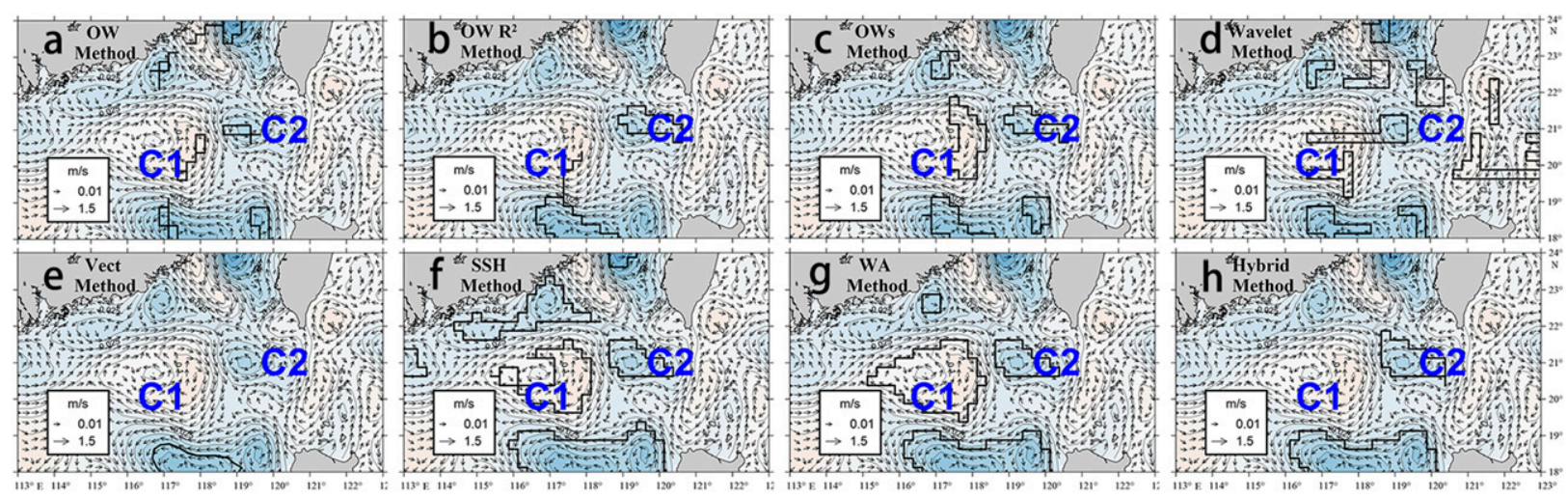

FIG. B4. As in Fig. B1, but for 9 Feb 2011 and the blue letter "C1" and "C2" in the ocean basin indicates the target eddies in this case study. 

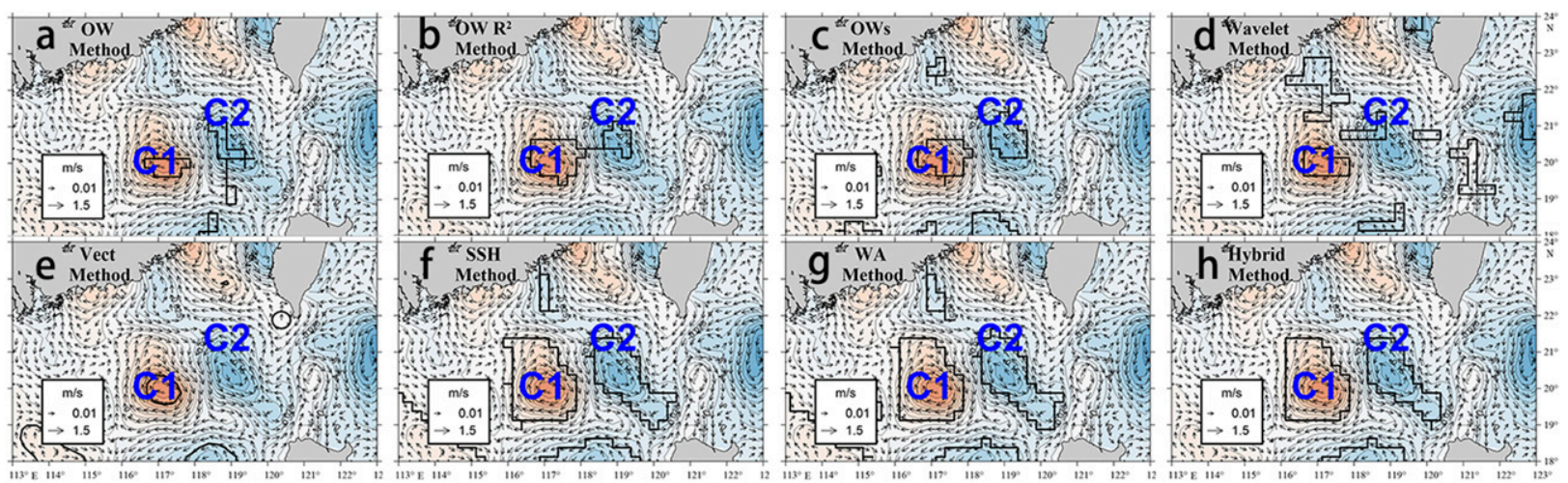

FIG. B5. As in Fig. B1, but for 7 Jan 2014 and the blue letter "C1" and "C2" in the ocean basin indicates the target eddies in this case study.

reason that abundant eddies exist in the western area of the LS might be related to the shedding of eddies from the loop of the Kuroshio or those locally generated (Nan et al. 2015; Zhang et al. 2017).

The second issue is about grouping phenomena of eddies in the SCS. A new perspective on the generation mechanism of eddies in the SCS has recently been proposed (Xie and Zheng 2017), and the authors considered the behaviors of eddies in the SCS to represent grouping phenomena and explained these events using the theory of Rossby normal modes. Based on this view, we sum the total number of the eddy trains (which are defined as eddy series consisting of more than three long-lived eddies whose distances with each other are less than $500 \mathrm{~km}$ ). Figure 11 presents the meridional distribution of the number of eddies that compose the eddy trains. The results from the OWs and OW methods are notably higher than the results obtained from the other methods. Additionally, all of the results can be divided into a crest and a
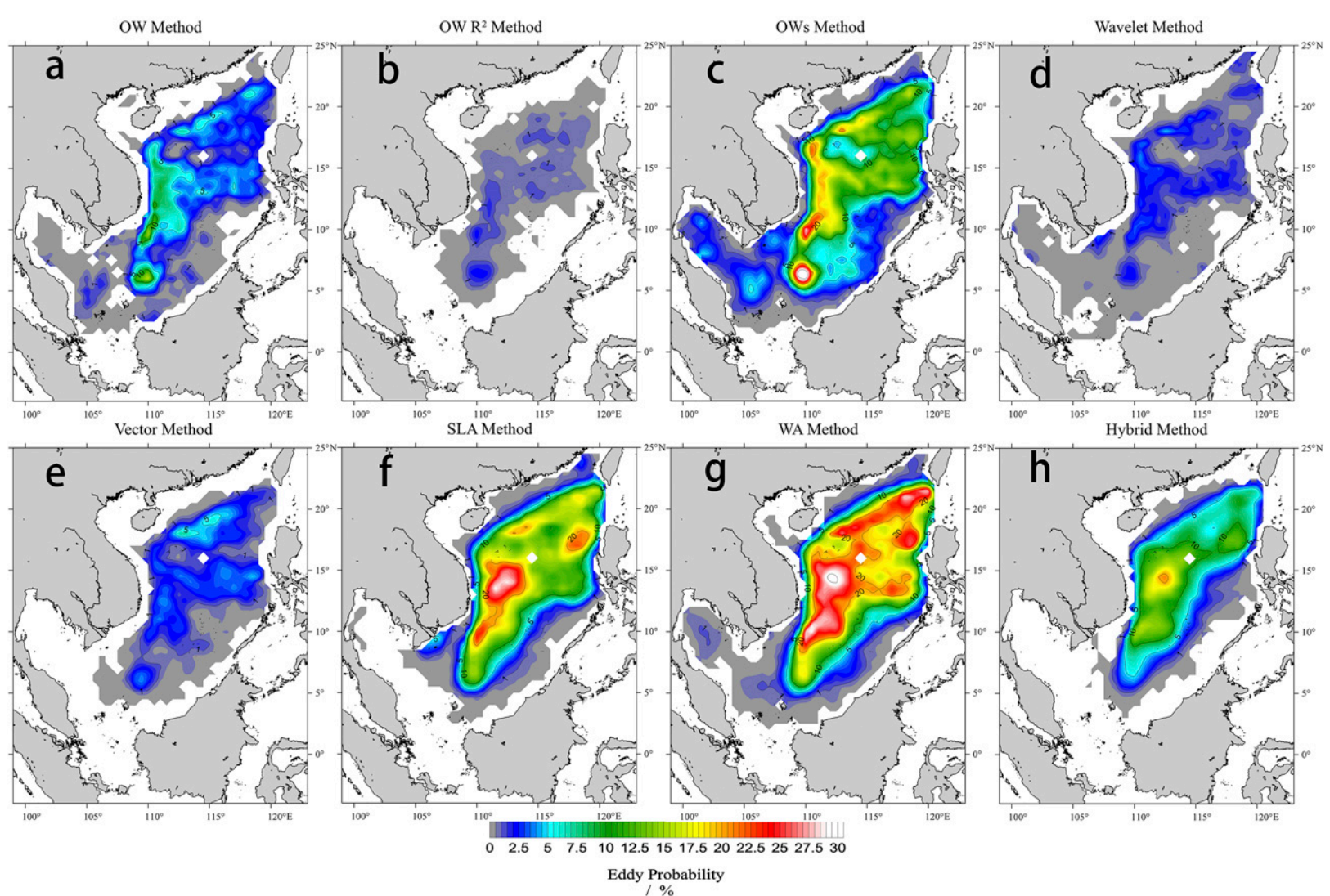

FIG. C1. Spatial distribution of the probability among all algorithms in Exp PB. 


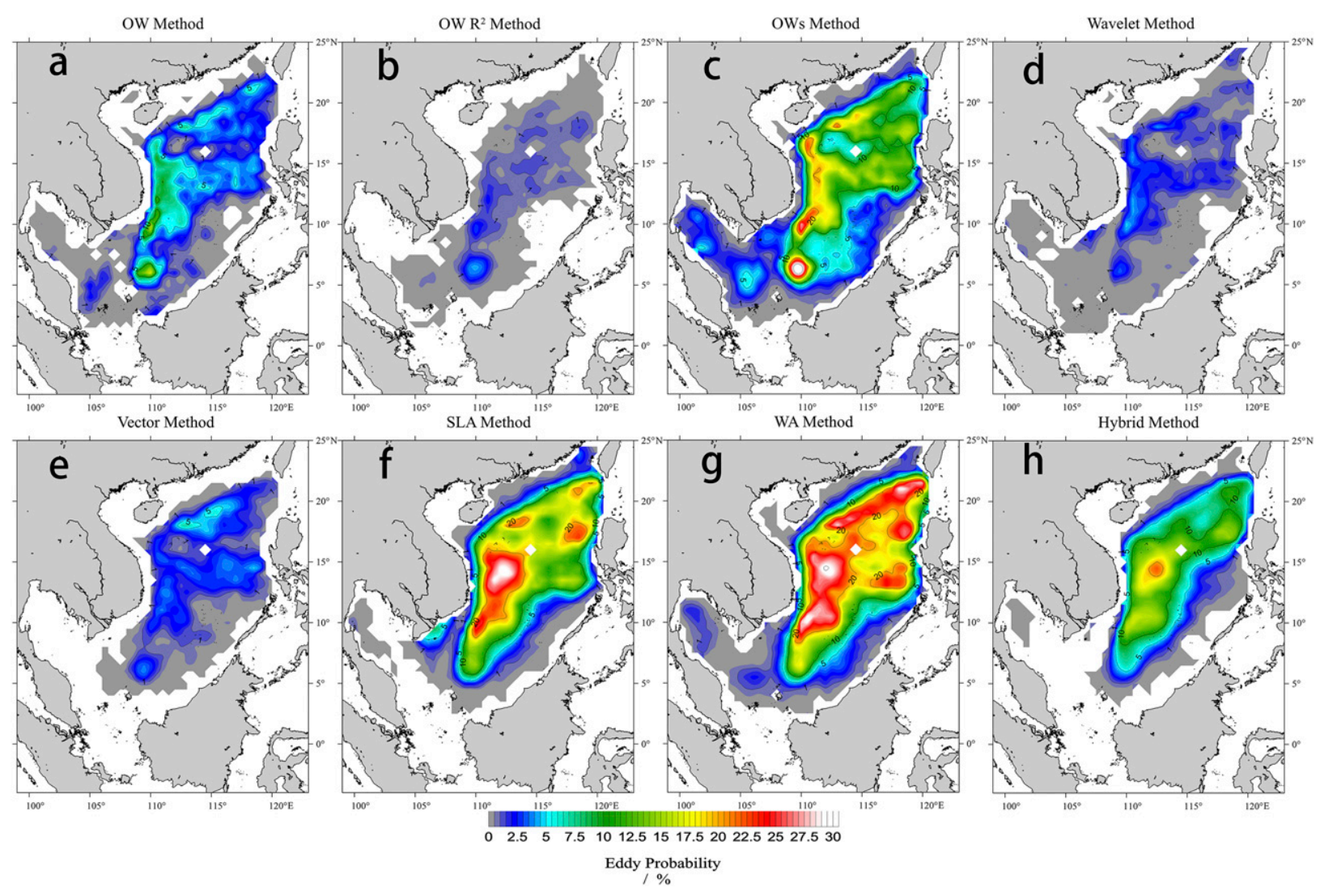

FIG. C2. As in Fig. C1, but in Exp PC.

trough of a wave (marked by the solid black horizontal lines in Fig. 11). The wavelength of this wave is approximately $16^{\circ}$ of latitude, which coincides with the theoretical meridional wavelength of the first mode of a standing wave, according to the theory proposed by Xie and Zheng (2017). There are notable subpeaks (denoted by the dashed black horizontal lines) embedded in the prominent waves. The interval between these subpeaks is approximately $4^{\circ}$ of latitude, which conforms to the second mode of a standing wave. These subpeaks are only obvious in the results of the OW and OWs methods and is hardly visible in the other results. This absence may be caused by the stronger attenuation signal in the southern part of the SCS, which is induced by the dissipation of energy during the propagation of the wave from the north to the south. Therefore, only the two most sensitive algorithms (OW and OWs) can detect signals in the southern SCS.

\section{Summary}

The present paper involves eight eddy detection algorithms. First, we assessed their performance through idealized experiments. All eddy identifications in the SCS were then compared and analyzed.

The discrepancy among all methods shown in the idealized experiments is very clear. Induced by the difference of the definition of the eddy area, the idealized experiments indicate that the class 1 algorithms underestimate the eddy areas. However, for a round eddy, their performances (except for the vector and wavelet methods) are better than the class 2 and 3 algorithms under high SNR. The class 1 algorithms cannot satisfactorily identify elliptical eddies.

In the SCS, the discrepancy among all methods is spatially variational and caused by different reasons. There are significant differences in the probabilities and areas of eddies among the eight eddy detection algorithms. The intensity of eddies have less discrepancy, and the differences in the lifetimes of eddies are negligible. Specifically, in terms of the eddy probabilities, based on the ensemble mean method, the maximum eddy probabilities are $18 \% \pm 11 \%$. Most of the zones with a high difference of eddy probabilities are of few eddies, except for the areas west of the LS, west of LI between $12^{\circ}$ and $17^{\circ} \mathrm{N}$, and at the southernmost end 


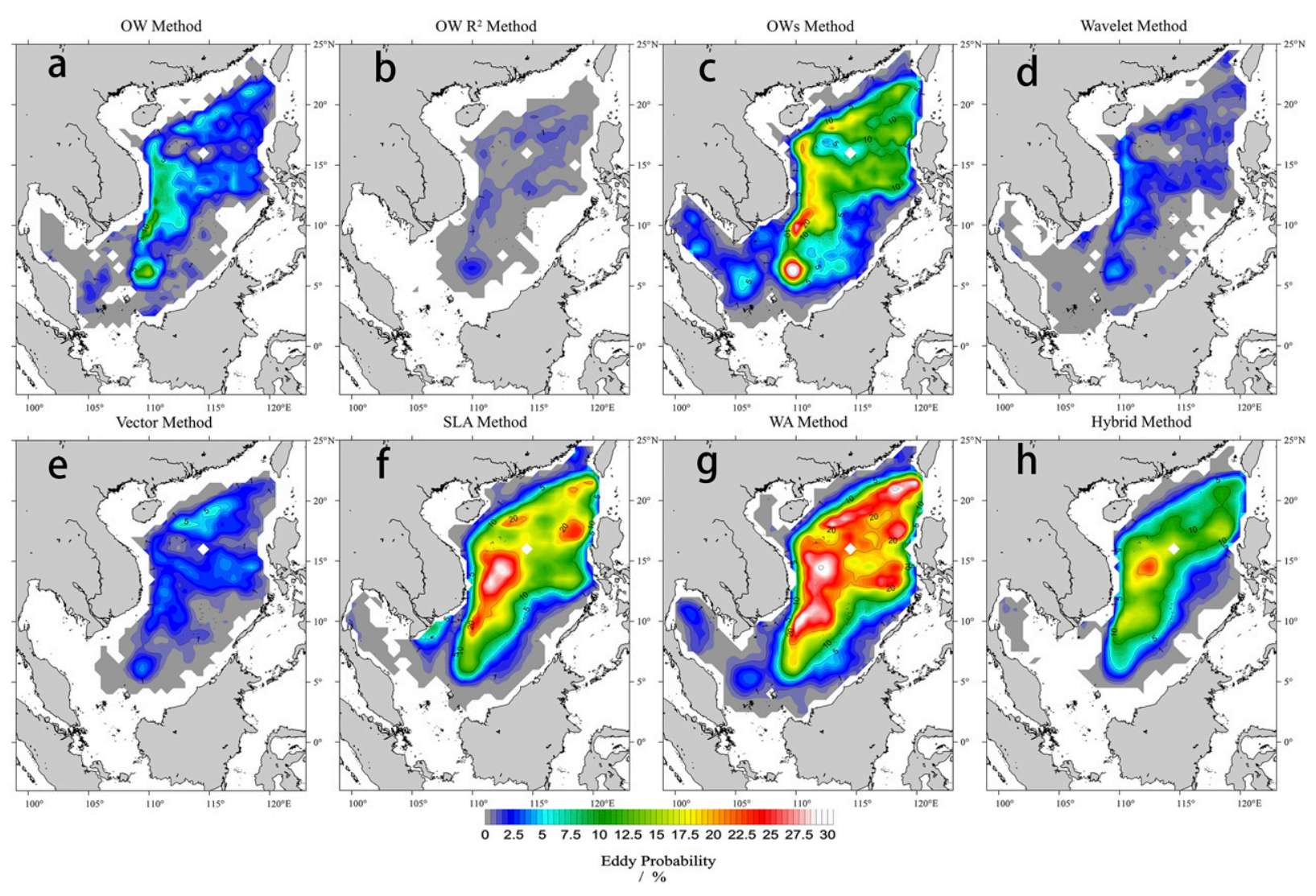

FIG. C3. As in Fig. C1, but in Exp PD.

of the SCS basin. They are caused by the relatively strong interferences, noncircular eddy shapes, and gentle SLA gradients produced by the geostrophic balance, respectively. Therefore, when carrying out eddy studies in these areas, we should pay particular attention to the impact of eddy detection algorithms. Through comparing the isolated eddy area with the baroclinic Rossby deformation radius, the results indicate that the SLA, WA, and hybrid methods extract more wavelike eddy features than the other algorithms. This can explain why the areas of structures isolated by these methods are larger near the Nansha Islands. In terms of the numbers of eddies that passed through the LS, the discrepancies among the algorithms are negligible. The grouping phenomena of eddies in the SCS are better reflected in the results of the OW and OWs methods.

Recently, the 3D structure of mesoscale eddies has become a hot topic for oceanographers (Chen et al. 2015; Wang et al. 2015; Zu et al. 2013). To detect an eddy in different vertical layers, the methods that aim to identify the close SLA isoline must be adjusted. The adjustment might include from the SLA isoline detection to the temperature, pressure, or other property isoline detection and the variational searching threshold along with the increasing depth. However, the methods that aim to identify swirling currents can be used to extract the 3D structure of a mesoscale eddy directly.

Last but not least, some strategic suggestions with regard to selecting suitable detection algorithms for studying oceanic mesoscale eddies in the SCS can be concluded:

(i) For a mean property study, deviations of the OW $R^{2}$ method are quite remarkable, and this method is not appropriate. The number of eddies obtained from the vector method tends to be underestimated, and it is overestimated using the wavelet method.

(ii) For a case study focusing on a "standard eddy," which has a high intensity and circular shape, the OW $R^{2}$ method might be viable. For a case study focusing on the peripheral area of an eddy, the WA, SLA, and hybrid methods are advisable.

(iii) From the perspective of the specific study location, in the southernmost SCS basin and west of LI and the LS, the OW and OWs methods are more sensitive. A target eddy can be easily detected by these methods. 


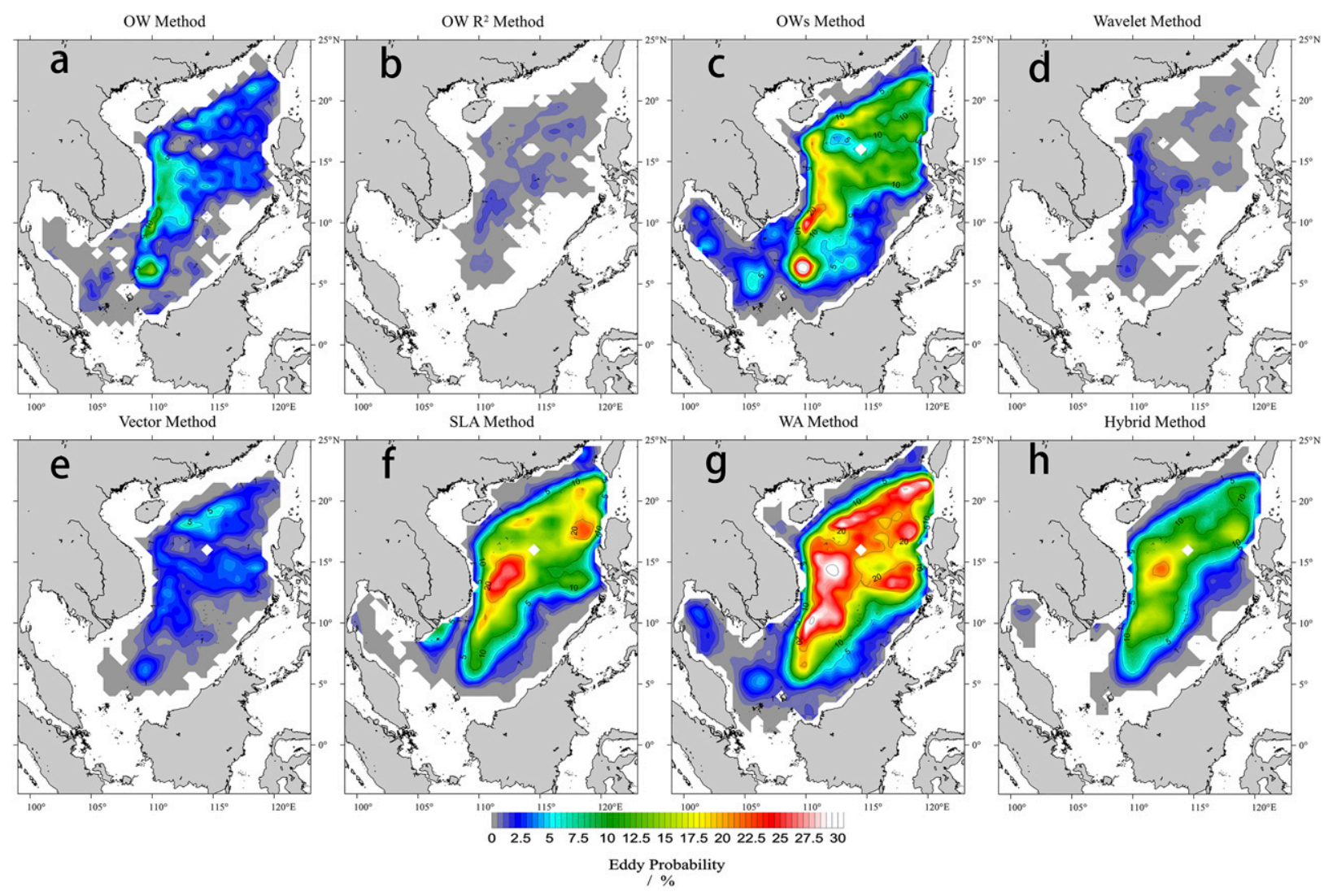

FIG. C4. As in Fig. C1, but in Exp PE.

In summary, no one method is perfect for all research purposes. For different studies, the most suitable method is different.

Acknowledgments. The authors thank Dr. Andrea M. Doglioli, Dr. Mark R. Petersen, and Dr. Changming Dong for providing the WATERS (wavelet method), OW $R^{2}$, and vector routines. The altimeter data were obtained from the AVISO website (http://aviso.oceanobs.com), and the temperature/salinity data (World Ocean Atlas 2013) were obtained from the NOAA website (https:// www.nodc.noaa.gov/OC5/woa13/woa13data.html). This work was supported by the National Natural Science Foundation of China (Grant 41506037), the Basic Scientific Fund for National Public Research Institutes of China (Grant GY0217Q06), the Natural Foundation of Shandong Province of China (Grant ZR2015PD009), and the NSFC-Shandong Joint

TABLE C4. As in Table C2, but for Exp PD.

\begin{tabular}{|c|c|c|c|c|}
\hline Detection algorithm & Probability (\%) & Intensity $(\mathrm{cm})$ & Area $\left(10^{4} \mathrm{~km}^{2}\right)$ & Lifetime (days) \\
\hline OW & 1.8 & 3.84 & 0.80 & 54.63 \\
\hline OWs & 5.2 & 4.32 & 1.61 & 64.42 \\
\hline OW $R^{2}$ & 0.3 & 12.12 & 2.98 & 37.62 \\
\hline Wavelet & 0.8 & 7.51 & 2.24 & 42.88 \\
\hline Vector & 1.3 & 2.38 & 2.31 & 44.32 \\
\hline SLA & 6.9 & 6.82 & 8.57 & 52.40 \\
\hline WA & 8.8 & 5.22 & 5.70 & 55.03 \\
\hline Hybrid & 4.6 & 8.32 & 10.61 & 48.18 \\
\hline Mean & 3.7 & 6.31 & 4.35 & 50.06 \\
\hline Standard deviation & 3.2 & 3.07 & 3.57 & 8.50 \\
\hline Coefficient of variation & 0.85 & 0.49 & 0.82 & 0.17 \\
\hline
\end{tabular}


TABLE C5. As in Table C2, but for Exp PE.

\begin{tabular}{lcccc}
\hline \hline Detection algorithm & Probability $(\%)$ & Intensity $(\mathrm{cm})$ & Area $\left(10^{4} \mathrm{~km}^{2}\right)$ & Lifetime $($ days $)$ \\
\hline OW & 1.7 & 3.82 & 0.78 & 1.62 \\
OWs & 5.2 & 4.32 & 3.12 & 65.02 \\
OW $R^{2}$ & 0.3 & 11.97 & 4.16 & 37.05 \\
Wavelet & 0.3 & 11.54 & 2.31 & 38.16 \\
Vector & 1.3 & 2.38 & 8.35 & 44.32 \\
SLA & 6.4 & 6.96 & 5.58 & 56.16 \\
WA & 8.7 & 5.06 & 11.56 & 54.28 \\
Hybrid & 4.4 & 8.89 & 4.69 & 50.02 \\
Mean & 3.6 & 6.87 & 3.67 & 49.83 \\
Standard deviation & 3.1 & 3.61 & 0.78 & 9.31 \\
Coefficient of variation & 0.87 & 0.53 & & 0.19 \\
\hline
\end{tabular}

Fund for Marine Science Research Centers (Grant U1606405).

\section{APPENDIX A}

\section{Eddy Identification under Specific Experiment Settings}

Eddy identification from all methods in Exp A with an SNR of 0.1 is shown in Fig. A1. Figure A2 shows similar results but from Exp B and with an ellipticity of 1.5.

\section{APPENDIX B}

\section{Eddy Identifications in Case Studies}

To provide more comparisons of the case study, we used all algorithms to extract eddies in specific time and locations. Each case refers to a previous study, and the references are as follows. Case 1 (Fig. B1) refers to Li et al. (1998), case 2 (Fig. B2) refers to Hu et al. (2011), case 3 (Fig. B3) refers to Nan et al. (2011), case 4 (Fig. B4) refers to Zhang et al. (2013), and case 5 (Fig. B5) refers to Zhang et al. (2016). The results in each case are different, but the general features of every algorithm are similar with the descriptions of the case (Fig. 10) in section $3 b$.

\section{APPENDIX C}

\section{A Parallel Experiment}

The parameters used in each algorithm can impact the final detections. We designed five experiments (Exps PA-PE) that each use different parameters. Exp PA is the experiment described in the manuscript. The inputs for Exp PB were selected to find the highest mean similarity in the objective experiment $\mathrm{B}(0<$ ellipticity $<0.5)$. The inputs for the tested algorithms in all experiments are listed in Table $\mathrm{C} 1$. Although the inputs are different, the general discrepancy among methods is consistent (Tables C2-C5; Figs. C1-C4). This suggests that the discrepancy is not parameter dependent and should be explained by the properties of mesoscale eddies in the SCS.

\section{REFERENCES}

Chaigneau, A., A. Gizolme, and C. Grados, 2008: Mesoscale eddies off Peru in altimeter records: Identification algorithms and eddy spatio-temporal patterns. Prog. Oceanogr., 79, 106-119, https://doi.org/10.1016/j.pocean.2008.10.013.

, G. Eldin, and B. Dewitte, 2009: Eddy activity in the four major upwelling systems from satellite altimetry (1992-2007). Prog. Oceanogr., 83, 117-123, https://doi.org/10.1016/j.pocean.2009.07.012.

Chelton, D. B., R. A. de Szoeke, M. G. Schlax, K. El Naggar, and N. Siwertz, 1998: Geographical variability of the first baroclinic Rossby radius of deformation. J. Phys. Oceanogr., 28, 433-460, https://doi.org/10.1175/1520-0485(1998)028<0433: GVOTFB $>2.0 . C O ; 2$.

—, M. G. Schlax, and R. M. Samelson, 2011: Global observations of nonlinear mesoscale eddies. Prog. Oceanogr., 91, 167-216, https://doi.org/10.1016/j.pocean.2011.01.002.

Chen, G., Y. Hou, and X. Chu, 2011: Mesoscale eddies in the South China Sea: Mean properties, spatiotemporal variability, and impact on thermohaline structure. J. Geophys. Res., 116, C06018, https://doi.org/10.1029/2010JC006716.

_ _ J. Gan, Q. Xie, X. Chu, D. Wang, and Y. Hou, 2012: Eddy heat and salt transports in the South China Sea and their seasonal modulations. J. Geophys. Res., 117, C05021, https:// doi.org/10.1029/2011JC007724.

— , and Coauthors, 2015: Observed deep energetic eddies by seamount wake. Sci. Rep., 5, 17416, https://doi.org/10.1038/ srep17416.

Chu, X., H. Xue, Y. Qi, G. Chen, Q. Mao, D. Wang, and F. Chai, 2014: An exceptional anticyclonic eddy in the South China Sea in 2010. J. Geophys. Res. Oceans, 119, 881-897, https://doi.org/ 10.1002/2013JC009314.

Doglioli, A. M., B. Blanke, S. Speich, and G. Lapeyre, 2007: Tracking coherent structures in a regional ocean model with wavelet analysis: Application to Cape Basin eddies. J. Geophys. Res., 112, C05043, https://doi.org/10.1029/2006JC003952.

Geng, W., Q. Xie, G. Chen, T. Zu, and D. Wang, 2016: Numerical study on the eddy-mean flow interaction between a cyclonic eddy and Kuroshio. J. Oceanogr., 72, 727-745, https://doi.org/ 10.1007/s10872-016-0366-0. 
,,,--- Q. Liu, and D. Wang, 2018: A three-dimensional modeling study on eddy-mean flow interaction between a Gaussian-type anticyclonic eddy and Kuroshio. J. Oceanogr., 74, 23-37, https://doi.org/10.1007/s10872-017-0435-z.

Guo, M., F. Chai, P. Xiu, S. Li, and S. Rao, 2015: Impacts of mesoscale eddies in the South China Sea on biogeochemical cycles. Ocean Dyn., 65, 1335-1352, https://doi.org/10.1007/ s10236-015-0867-1.

Henson, S. A., and A. C. Thomas, 2008: A census of oceanic anticyclonic eddies in the Gulf of Alaska. Deep-Sea Res. I, 55, 163-176, https://doi.org/10.1016/j.dsr.2007.11.005.

Hu, J., Z. Gan, J. Zhu, and M. Dai, 2011: Observed three dimensional structure of a cold eddy in the southwestern South China Sea. J. Geophys. Res., 116, C05016, https://doi.org/ 10.1029/2010JC006810.

Li, L., W. D. Nowlin, and S. Jilan, 1998: Anticyclonic rings from the Kuroshio in the South China Sea. Deep-Sea Res. I, 45, 14691482, https://doi.org/10.1016/S0967-0637(98)00026-0.

Lin, X., C. Dong, D. Chen, Y. Liu, J. Yang, B. Zou, and Y. Guan, 2015: Three-dimensional properties of mesoscale eddies in the South China Sea based on eddy-resolving model output. DeepSea Res. I, 99, 46-64, https://doi.org/10.1016/j.dsr.2015.01.007.

Nan, F., Z. He, H. Zhou, and D. Wang, 2011a: Three long-lived anticyclonic eddies in the northern South China Sea. J. Geophys. Res., 116, C05002, https://doi.org/10.1029/2010JC006790.

$\longrightarrow$, H. Xue, P. Xiu, F. Chai, M. Shi, and P. Guo, 2011b: Oceanic eddy formation and propagation southwest of Taiwan. J. Geophys. Res., 116, C12045, https://doi.org/10.1029/2011JC007386.

,-- , and F. Yu, 2015: Kuroshio intrusion into the South China Sea: A review. Prog. Oceanogr., 137, 314-333, https:// doi.org/10.1016/j.pocean.2014.05.012.

Nencioli, F., C. Dong, T. Dickey, L. Washburn, and J. McWilliams, 2010: A vector geometry-based eddy detection algorithm and its application to a high-resolution numerical model product and high-frequency radar surface velocities in the Southern California Bight. J. Atmos. Oceanic Technol., 27, 564-579, https://doi.org/10.1175/2009JTECHO725.1.

Petersen, M. R., S. J. Williams, M. E. Maltrud, M. W. Hecht, and B. Hamann, 2013: A three-dimensional eddy census of a highresolution global ocean simulation. J. Geophys. Res. Oceans, 118, 1759-1774, https://doi.org/10.1002/jgrc.20155.

Sadarjoen, I. A., and F. H. Post, 2000: Detection, quantification, and tracking of vortices using streamline geometry. Comput. Graphics, 24, 333-341, https://doi.org/10.1016/S0097-8493(00)00029-7.

Sheu, W.-J., C.-R. Wu, and L.-Y. Oey, 2010: Blocking and westward passage of eddies in the Luzon Strait. Deep-Sea Res. II, 57, 1783-1791, https://doi.org/10.1016/j.dsr2.2010.04.004.

Souza, J. M. A. C., C. Montégut, and P. Y. Traon, 2011: Comparison between three implementations of automatic identification algorithms for the quantification and characterization of mesoscale eddies in the South Atlantic Ocean. Ocean Sci., 7, 317-334, https://doi.org/10.5194/os-7-317-2011.

Wang, D., H. Xu, J. Lin, and J. Hu, 2008: Anticyclonic eddies in the northeastern South China Sea during winter 2003/2004. J. Oceanogr., 64, 925-935, https://doi.org/10.1007/s10872-008-0076-3.

Wang, G., J. Su, and P. C. Chu, 2003: Mesoscale eddies in the South China Sea observed with altimeter data. Geophys. Res. Lett., 30, 2121, https://doi.org/10.1029/2003GL018532.

Wang, Q., L. Zeng, W. Zhou, Q. Xie, S. Cai, J. Yao, and D. Wang, 2015: Mesoscale eddies cases study at Xisha waters in the South China Sea in 2009/2010. J. Geophys. Res. Oceans, 120, 517-532, https://doi.org/10.1002/2014JC009814.

Williams, S., M. Petersen, P. T. Bremer, M. Hecht, V. Pascucci, J. Ahrens, M. Hlawitschka, and B. Hamann, 2011: Adaptive extraction and quantification of geophysical vortices. IEEE Trans. Visualization Comput. Graphics, 17, 2088-2095, https:// doi.org/10.1109/TVCG.2011.162.

Xie, L., and Q. Zheng, 2017: New insight into the South China Sea: Rossby normal modes. Acta Oceanol. Sin., 36, 1-3, https:// doi.org/10.1007/s13131-017-1077-0.

Xiu, P., F. Chai, L. Shi, H. Xue, and Y. Chao, 2010: A census of eddy activities in the South China Sea during 19932007. J. Geophys. Res., 115, C03012, https://doi.org/10.1029/ 2009JC005657.

Yi, J., Y. Du, Z. He, and C. Zhou, 2014: Enhancing the accuracy of automatic eddy detection and the capability of recognizing the multi-core structures from maps of sea level anomaly. Ocean Sci., 10, 39-48, https://doi.org/10.5194/os-10-39-2014.

Yuan, D., W. Han, and D. Hu, 2007: Anti-cyclonic eddies northwest of Luzon in summer-fall observed by satellite altimeters. Geophys. Res. Lett., 34, L13610, https://doi.org/10.1029/ 2007 GL029401.

Zhang, Y., Z. Liu, Y. Zhao, W. Wang, J. Li, and J. Xu, 2014: Mesoscale eddies transport deep-sea sediments. Sci. Rep., 4, 5937, https://doi.org/10.1038/srep05937.

Zhang, Z., W. Zhao, J. Tian, and X. Liang, 2013: A mesoscale eddy pair southwest of Taiwan and its influence on deep circulation. J. Geophys. Res. Oceans, 118, 6479-6494, https://doi.org/ 10.1002/2013JC008994.

_- J. Tian, B. Qiu, W. Zhao, P. Chang, D. Wu, and X. Wan, 2016: Observed 3D structure, generation, and dissipation of oceanic mesoscale eddies in the South China Sea. Sci. Rep., 6, 24349 , https://doi.org/10.1038/srep24349.

_ - W. Zhao, B. Qiu, and J. Tian, 2017: Anticyclonic eddy sheddings from Kuroshio loop and the accompanying cyclonic eddy in the northeastern South China Sea. J. Phys. Oceanogr., 47, 1243-1259, https://doi.org/10.1175/JPO-D-16-0185.1.

Zu, T., D. Wang, C. Yan, I. Belkin, W. Zhuang, and J. Chen, 2013: Evolution of an anticyclonic eddy southwest of Taiwan. Ocean Dyn., 63, 519-531, https://doi.org/10.1007/s10236-013-0612-6. 